\title{
Warburg, lecteur de Semper : ornement, parure et analogie cosmique
}

\section{Spyros Papapetros}

Traducteur : Marie Sanquer et Anika Schwarzwald

\section{OpenEdition \\ Journals}

Édition électronique

URL : http://journals.openedition.org/imagesrevues/2862

DOI : 10.4000/imagesrevues.2862

ISSN : 1778-380

\section{Éditeur :}

Centre d'Histoire et Théorie des Arts, Groupe d'Anthropologie Historique de l'Occident Médiéval, Laboratoire d'Anthropologie Sociale, UMR 8210 Anthropologie et Histoire des Mondes Antiques

\section{Référence électronique}

Spyros Papapetros, «Warburg, lecteur de Semper : ornement, parure et analogie cosmique », Images Re-vues [En ligne], Hors-série 4 | 2013, mis en ligne le 30 janvier 2012, consulté le 30 janvier 2021. URL : http://journals.openedition.org/imagesrevues/2862 ; DOI : https://doi.org/10.4000/ imagesrevues.2862

Ce document a été généré automatiquement le 30 janvier 2021.

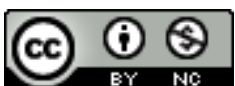

Images Re-vues est mise à disposition selon les termes de la Licence Creative Commons Attribution Pas d'Utilisation Commerciale 4.0 International. 


\section{Warburg, lecteur de Semper : ornement, parure et analogie cosmique}

\section{Spyros Papapetros}

Traduction : Marie Sanquer et Anika Schwarzwald

1 Entouré d'un monde plein de merveilles et de forces - et il a conscience des lois de ce monde qu'il s'efforce de résoudre sans jamais éclaircir, ces lois qui se précipitent vers lui sous la forme d'harmonies fragmentaires individuelles et qui maintiennent ses sentiments dans un état continu de surexcitation inassouvie l'homme évoque la perfection qui lui échappe à travers le jeu ; il construit pour lui-même un monde miniature dans lequel les lois cosmiques apparaissent sous leur forme la plus étroite et la plus compacte, mais renfermées en ellesmêmes, et, à cet égard, parfaites. Dans ce jeu, l'homme satisfait son instinct cosmogonique. ${ }^{1}$

Dans ce passage célèbre des Prolegomena

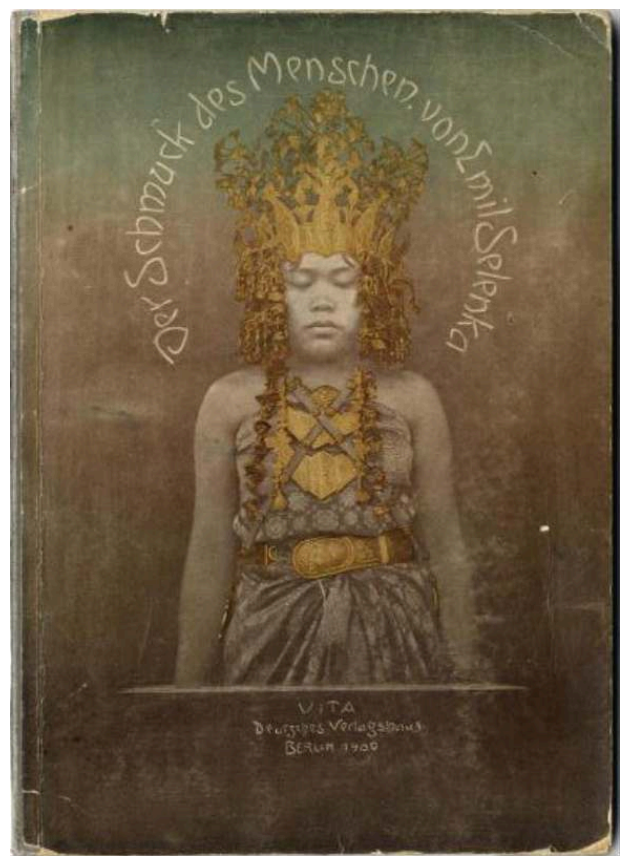
de son œuvre imposante, Der Stil, Gottfried Semper transforme les objets humains en modèles miniatures du monde et de ses principes de fonctionnement impénétrables. L'homme ne peut jamais tout à fait comprendre le monde, mais il peut élaborer des modèles pour l'aider à le reproduire et révéler, par analogie, toutes les opérations cosmiques. Chaque objet fabriqué par l'homme peut schématiser et connoter symboliquement ces relations analogiques dans 
la société et dans la nature: c'est avant tout un ornement. Si l'imagination anthropomorphique ou zoomorphique sert de médiateur entre les correspondances cosmologiques pendant l'Antiquité et la Renaissance, dans le monde post-newtonien de Semper, les mêmes analogies sont transformées en axes géométriques, qui soutiennent à leur tour la mécanique céleste de l'univers. Les flèches, les vecteurs et les lignes diagonales qui indiquent les forces physiques remplacent (ou croisent) les anciens personnages allégoriques - les Muses ou les Putti amoureuses - dont les corps symétriques masquaient, en tant qu'ornement, et démontraient en même temps, les lois de l'harmonie cosmique. Afin de comprendre le monde, l'homme, sans en avoir nécessairement conscience, grave l'univers à la surface des objets, mais il l'inscrit aussi rationnellement en diagrammes scientifiques, qui peuvent eux-mêmes devenir des ornements par la suite. Une telle transition dans l'iconographie et la performance épistémologique des ornements vers un système moins transparent de références se manifeste graphiquement dans les notes du lecteur de Semper le plus perspicace mais malheureusement peu reconnu, Aby Warburg, un historien de l'art de la Renaissance de la fin du XIXe siècle, dont les oreilles résonnaient toujours de ce que Semper appelle les « harmonies fragmentaires » produites par l'ornementation.

\section{Notes ornementées}

Un ensemble d'axes perpendiculaires représentant les quatre points cardinaux, désignés par une majuscule; une flèche horizontale pointant vers la gauche, à coté d'une ligne verticale; un rectangle avec deux lignes en pointillés, et dont la diagonale est également en pointillé ; plus bas, le petit dessin d'un arbre traversé par un axe symétrique; et encore en-dessous, la forme minuscule d'un homme aux bras partiellement ouverts (qui rappelle les représentations chrétiennes de la résurrection). (Fig. 1) 
Fig.1

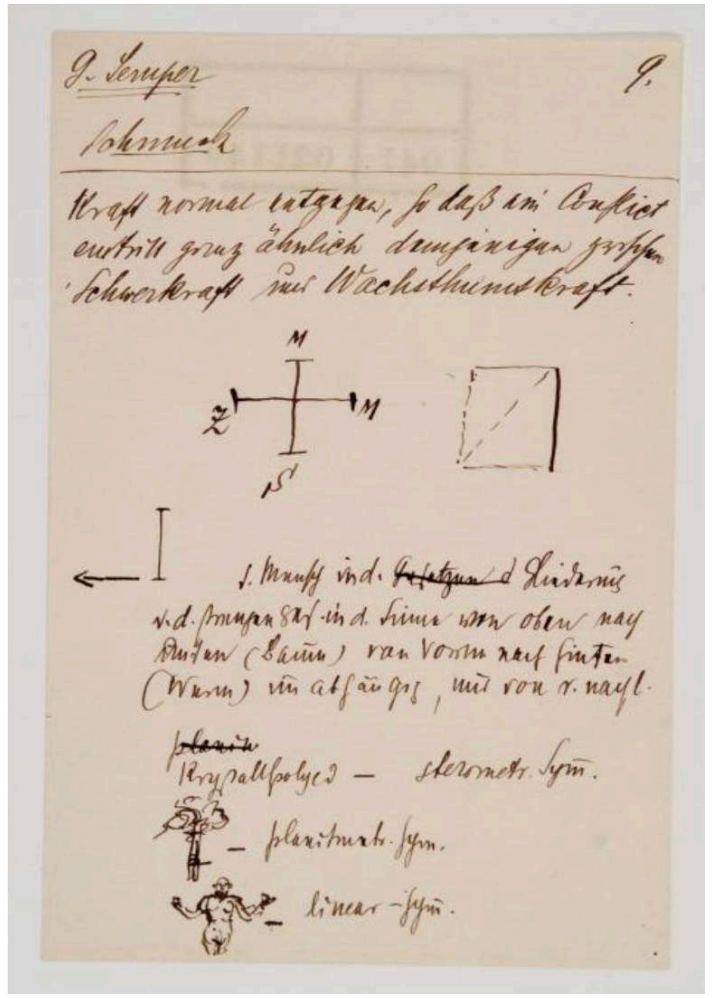

Aby Warburg, notes manuscrites et esquisses sur l'essai de Gottfried Semper de 1856 "Über die formelle Gesetzmässigkeit des Schmuckes und dessen Bedeutung als Kunstsymbol," Warburg Institute Archive, Zettelkasten Aesthetik WIA, ZK 041/ 021149. Photo: Warburg Institute Archive, London.

4 Ces cinq dessins se trouvent sur une des petites fiches de lecture de Warburg remplies de notes manuscrites sur Gottfried Semper - et plus particulièrement sur la conférence de l'architecte de 1856 "Sur les principes formels de la parure et leur signification comme symboles dans l'art $»^{2}$, et sur les Prolegomena de son Der Stil, dont le passage sur "l'instinct cosmogonique de l'homme » a déjà été cité au début de cette étude. ${ }^{3}$ (Fig.2) 


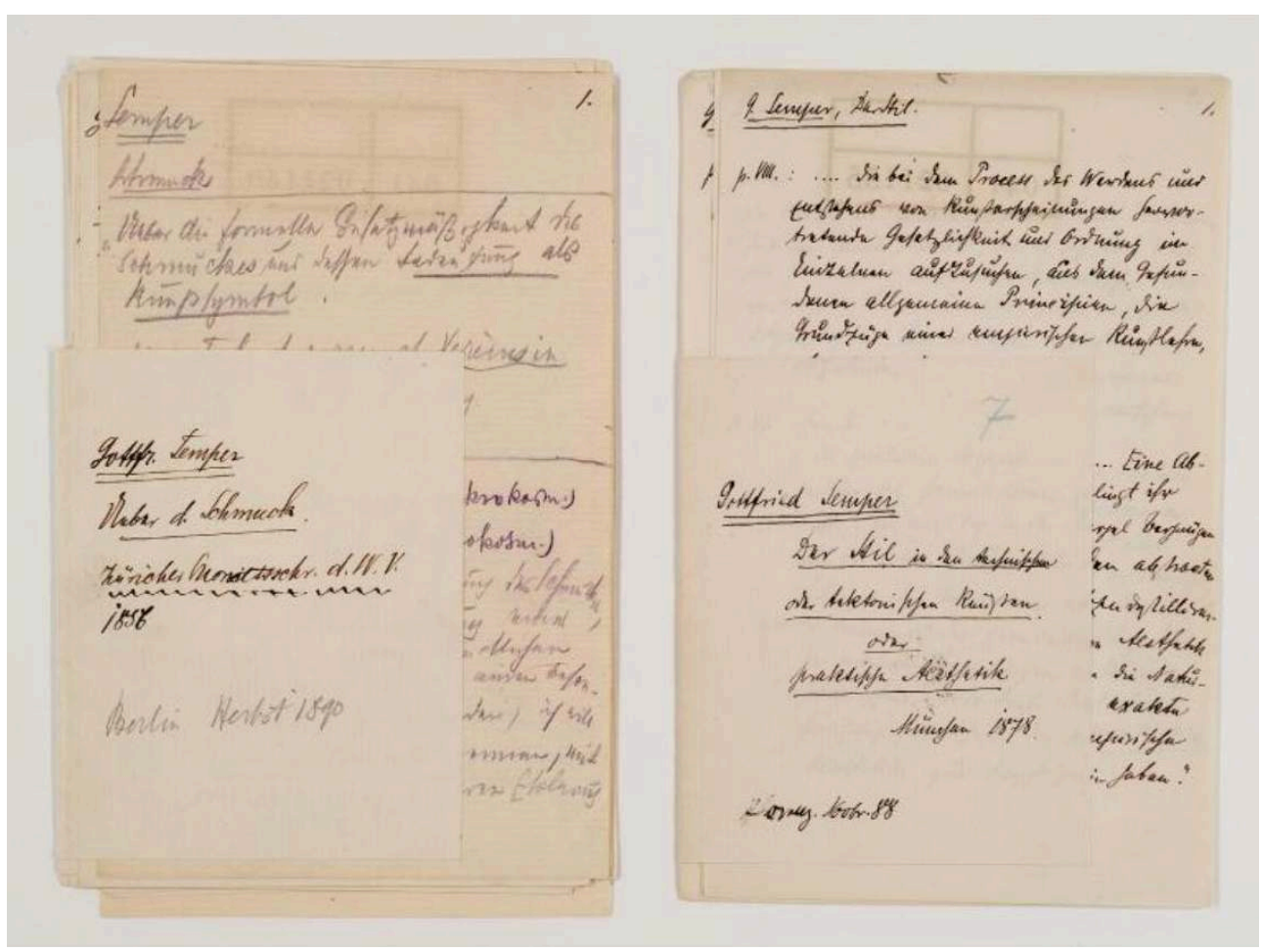

Aby Warburg, notes manuscrites sur l'essai de Gottfried Semper de 1856 "über d. [en] Schmuck" (notes datées de "Berlin, automne 1890") et Der Stil in den technischen und tektonischen Künsten (notes datées de "Florence, novembre 1888") Warburg Institute Archive, Zettelkasten Aesthetik WIA, ZK 041/ 021149 Warburg Institute Archive, ZK 041/021140-158. Photo: Warburg Institute Archive, London

5 La conférence sur la parure de Semper a été publiée sans illustrations. Peut-être qu'arrivé à un passage où le développement théorique sur la forme devenait particulièrement abstrait, Warburg (qui n'étais alors qu'un jeune étudiant en histoire de l'art) a-t-il ressenti le besoin de l'illustrer par ses propres dessins. Ses notes manuscrites sur le texte de Semper indiquent que les quatre axes en haut de la page représentent deux paires de forces cosmiques opposées: "la force de gravité" (Schwerkraft) et "la force de croissance" (Wachtumskraft) d'un côté, et le mouvement et la résistance au mouvement de l'autre. Semper conçoit ces quatre axes comme rayonnant à partir de "quatre centres" combinés en "deux paires." ${ }^{4}$ La flèche horizontale inscrite en dessous de ce diagramme suggère peut-être la direction de la résistance, qui est perpendiculaire à l'axe de croissance. Pour finir, le produit formel de la lutte entre le mouvement et la croissance est représenté, chez Warburg, par le croquis du petit arbre, une entité qui, selon Semper, révèle une symétrie "planimétrique" [sur plusieurs plans], qui contraste avec la symétrie "linéaire" de l'homme. L'homme est le seul être vivant qui se déplace perpendiculairement à son axe de croissance, écrit Semper, à la différence du poisson, par exemple, ou du "ver" (Wurm) qui se déplace "d'avant en arrière" (von vorne nach hinten) comme le mentionne Warburg dans ses notes. ${ }^{5}$

6 L'ensemble des dessins de Warburg détermine un modèle de transformation cosmique. Comme l'homme déconcerté décrit dans le prologue de Der Stil, l'historien de l'art a du mal à saisir l'univers théorique abstrait de l'architecte, c'est pourquoi il le convertit en modèle graphique qui représente en miniature les principes cosmiques de la 
morphologie et de la physique. Une fois de plus, l'orientation devient ornementation, et les lois de la symétrie cosmique de Semper se transforment en bijou graphique et réfléchissant.

7 Il est révélateur que le terme principal utilisé par Semper pour connoter la décoration corporelle et architecturale tout au long de sa conférence de 1856 ne soit pas « ornement » mais "parure" [Schmuck], un objet concret servant surtout à la mise en valeur du corps, comme un bijou, un collier ou une coiffure - des articles de parure plutôt destinés aux femmes, bien que les hommes en portent dans d'autres cultures et à d'autres époques. Selon Semper, les débats autour de l'ornementation ne devraient pas avoir trait à des formes géométriques désincarnées, mais à des objets qui ont un poids et des dimensions physiques, des objets qui s'inscrivent dans le monde par leur mouvement et leur orientation.

8 Semper inaugure sa conférence par la définition étymologique du mot Grec, Kosmos (également retranscrite par Warburg sur la première page de ses notes), un mot qui signifie à la fois ornementation et ordre du monde (Welt Ordnung). ${ }^{6}$ La source de Semper pourrait venir du Kosmos d'Humboldt, une histoire universelle du monde écrite par le physicien après son séjour en Amérique, dont le premier volume comprend un excursus de deux pages sur la sémasiologie du mot grec.7 Cependant l'architecte pourrait aussi avoir consulté directement des sources anciennes, car sa bibliothèque contenait de nombreux ouvrages d'auteurs grecs. Le lexique grec de 1819 de Schneider, qui se trouvait dans cette bibliothèque, interprète le Kosmos comme "parure" (Schmuck), "ordre" (Ordnung), "hiérarchie" (Anordnung), ainsi que "direction" (Einrichtung) et "discipline" (Disciplin). ${ }^{8}$ D'autres dictionnaires contemporains, tel que le célèbre manuel de Passow, donne à ce mot une pléthore de significations, d'"honnneur," "louange" et "attribution" (Zier, Ehre, Lob, Auszeichnung), à "parure pour les chevaux" (Pferdeschmuck). ${ }^{9}$ Toutefois pour Semper, le Kosmos relève avant tout de trois mondes distincts, de trois disciplines cosmiques, différentes mais parallèles, qui convergent finalement en un seul système épistémologique. ${ }^{10}$

9 Le premier de ces mondes est celui de la philosophie antique, source des étymologies grecques de Semper (qui, même si elles ne sont pas toujours exactes, sont toujours prémonitoires par leurs associations). C'est le monde des anciennes Victoires et des Grâces, des couronnes décoratives et des acrotères attiques duquel il tire son vocabulaire des formes décoratives. Mais ce monde ancien rétrograderait peu à peu à un arrière-plan allégorique et courrait le risque de devenir incompréhensible, seulement ornemental, s'il n'était pas soutenu par le deuxième monde de l'architecte.

10 Ce deuxième monde, c'est celui des sciences naturelles et de la physique. Ce monde éclectique est un mélange de l'astrophysique universelle de Newton et Lagrange et de la philosophie naturelle de Schopenhauer, que Warburg a schématisés dans ses esquisses. C'est le monde des queues de comètes et des projectiles de frondes antiques, dont Semper a étudié la trajectoire et la forme pendant son exil britannique, en se basant sur ses études universitaires en physique et en mathématique. ${ }^{11}$ Sa conférence sur l'ornementation lui a donné une nouvelle occasion de montrer que les principes cosmiques, schématisés par des objets volant bien plus haut que la surface de la terre, avaient également informé la conception de plus petits objets qui évoluent beaucoup plus près de celle-ci. Que ce soit un missile ou un bijou, la forme même d'un objet bien conçu peut, pour Semper, restaurer l'ordre et la légitimité dans un monde qui semble ne plus en avoir. 
11 À coté des perspectives cosmiques offertes par la philologie et la physique, un troisième monde s'impose à Semper, c'est celui de l'ethnographie. ${ }^{12}$ Son expérience en tant que désignateur à l'Exposition Internationale de 1851 au Crystal Palace à Londres - luimême un microcosme architectural, dont chaque salle est dédiée à une nation différente - lui procure un inventaire ethnologique des formes décoratives, ainsi qu'un modèle méthodologique pour faire fusionner la variété des cultures en une seule structure ornée. ${ }^{13}$ Selon Semper, l'ethnographie permet de compléter les théories architecturales et cosmiques de Vitruvius et les prémices épistémologiques de la science moderne, en prouvant qu'en fin de compte, l'homme a plus d'un axe de symétrie, son orientation s'en trouve alors reconfigurée au-delà des limites de l'ordre classique ${ }^{14}$ Ce n'est pas par hasard si l'un des premiers exemples de parures que Semper a choisi pour sa conférence est celui des Botocudos de l'Est du Brésil car elles sont déjà décrites par plusieurs ethnologues dans leurs ouvrages. ${ }^{15}$ Les Botocudos, explique Semper, étirent leurs lèvres inférieures et leurs lobes d'oreille à “ des longueurs étonnantes" en y introduisant "des morceaux de bois, des os, des coquillages et d'autres objets du même genre."16 Les théoriciens de l'empathie décrivent souvent les objets décoratifs comme des prolongements imaginaires du corps. Pourtant, pour les Botocudos, l'objet ajouté n'est pas une extension mais plutôt une insertion ; le corps lui-même doit prolonger et littéralement intégrer l'objet. L'origine de ce que Semper appelle "la pulsion de décoration" (Verzierungstrieb) ne réside de ce fait pas dans le plaisir et l'empathie autosatisfaisante, mais plutôt dans l'étirement douloureux du corps au-delà des limites naturelles.

12 A un autre moment révélateur de sa conférence, Semper avance que les lèvres percées et les pendentifs en os massifs des Botocudos peuvent être considérés comme "les premiers rudiments [die erste Rudimente]" des "boucles d'oreilles" qui rehaussaient les beautés helléniques et qui ont continué à être appréciées jusqu'à récemment, avant de tomber en disgrâce à cause de la mode contemporaine. ${ }^{17}$ C'est comme si l'ethnographie avait retrouvé les rudiments originaux de la fonction symbolique à la surface de la parure moderne. La tâche que se donne alors l'architecte est de recombiner les vecteurs tracés par les trois disciplines - ethnographie, philologie et physique - pour former un modèle composite schématisant les dynamiques actuelles du monde.

\section{Les trois catégories de la parure}

L'aspect "impressionnant" des parures Botocudos, et les scarifications des sociétés tribales ont, selon l'architecte, une qualité "d'informe" (formloss) ou "d'inarticulé" (ungeglierte). ${ }^{18}$ Ils représentent une étape prélinguistique dans l'évolution de la parure qui ne se transformera que bien plus tard en langage universel aux motifs décoratifs plus réguliers. "I "Il semble qu'un sentiment naturel ait conduit tous les peuples à adopter des formes analogues," déclare Semper en se référant à une certaine forme de casques: "Le casque de l'habitant de la Nouvelle-Zélande est presque identique avec celui des Grecs des premiers âges." ${ }^{20}$ C'est en se basant sur ces ressemblances considérables et en s'appuyant sur l'ethnographie, la philologie, et la physique, que Semper classe les ornements en trois catégories structurelles, en fonction de la forme de mouvement cosmique décrit par chaque type. Dans ses notes, Warburg commente abondamment chaque catégorie. 
14 La première catégorie est celle du pendentif (Behang). Cet objet, dont l'une des extrémités est souvent libre, se caractérise par son oscillation, comme, par exemple, une boucle d'oreille ou un médaillon suspendu à une chaîne. Parce qu'il est en suspension, le pendentif manifeste la loi cosmique de la pesanteur, il est par conséquent considéré comme un ornement "macrocosmique." ${ }^{21}$ Le pendentif incarne l'oscillation entre mouvement et équilibre, il souligne aussi la symétrie, c'est pourquoi il apparait souvent en paires. En dehors des bijoux, Semper classe parmi les pendentifs, les pans de tissus des vêtements, puisqu'ils tombent vers la terre et pendent symétriquement, ainsi que certaines coiffures, telles que les longues tresses des Grecs et les barbes des Assyriens. ${ }^{22}$ Warburg, quant à lui, reconfigure draperies et coiffures comme incarnant l'équilibre ou de l'instabilité psychologique, à cause de leur caractère oscillatoire tantôt régulier, tantôt irrégulier.

Semper avait déjà prédit ce tournant psychologique dans la fonction de l'ornement. D'un côté, de par sa verticalité, le pendentif possède une valeur esthétique, il permet d'accentuer les courbures du visage, du cou, et d'autres parties du corps; d'un autre côté, il a aussi une valeur psychologique car il extériorise le caractère et le tempérament de la personne qui le porte. ${ }^{23}$ Par exemple, les boucles d'oreilles d'une femme nerveuse se déplaceraient spasmodiquement et oscilleraient trop. Le pendentif mesure et modère le mouvement interne et externe de celui qui le porte, et traduit le décorum dans la décoration. Avec ses oscillations périodiques, le pendentif sert d'intermédiaire entre la physiologie cosmique et la psychologie personnelle. Quelques décennies après Semper, Warburg élargit la circonférence du pendentif de la psychologie à l'épistémologie, et redéfinit l'ensemble de ses oscillations en idéogramme de la variation périodique entre l'équilibre et l'agitation. Le pendentif devient alors le symbole même de la polarité entre des systèmes épistémologiques concurrents, tels que la magie païenne et la science moderne.

16 Le deuxième genre d'ornement selon Semper est l'anneau. Il comprend les colliers, les anneaux passés à l'oreille, aux doigts et aux orteils, ainsi que les couronnes et les bandeaux pour la tête, le genou, les bras ou la poitrine. L'anneau met en valeur les proportions du corps. Si le pendentif rattache le corps au macrocosme, l'anneau joue un rôle plus microcosmique, il délimite le rapport qu'entretient chaque partie du corps avec les autres. ${ }^{24}$ Les ornements en forme d'anneau tels que les bandeaux sur les bras ou les jambes peuvent aussi fournir un support ou renforcer la vaillance et le courage. Semper évoque notamment "les bracelets d'or" des jeunes Cafres qui, "à mesure que les membres se développent, entrent profondément dans les chairs" pour promouvoir la croissance musculaire. ${ }^{25}$

En plus d'être le siège du pouvoir physique, l'anneau peut conférer ou accentuer le pouvoir social, et même politique, de celui qui le porte. Selon Semper, l'ornement en forme d'anneau apparaît toujours dans une configuration radiale et rythmique, il dirige toute l'attention sur la partie du corps qu'il encercle. Pour cette raison, les ornements en forme d'anneau les plus prestigieux sont ceux qui entourent la tête, les bandeaux et les couronnes des rois et autres dirigeants. ${ }^{26}$ L'anneau attire toute l'attention à soi, mais, par un jeu de réflexion, il peut aussi renvoyer cette énergie dans le monde. C'est le pivot des relations centripètes et centrifuges, il fonctionne donc comme le symbole des formations sociales.

Tandis qu'il semble être statique, l'ornement annulaire dispose d'un fort potentiel de mouvement virtuel. Un collier, par exemple, peut s'étendre au-delà de sa périphérie 
restreinte pour en arriver à couvrir une plus grande surface du corps, comme dans le cas des plastrons égyptiens mentionnés par Semper dans son Der Stil. L'ajout d'un ou de plusieurs pendentifs accentue encore le caractère expansif des objets en forme d'anneau, Semper prend l'exemple du Moyen-âge où l'on attachait autour de sa taille, sur des chaines ou des ceintures des clés, des épées, des croix, et des petits livres de prières.$^{27}$ L'anneau peut même être considéré comme étant à l'origine de l'habillement, car dans certaines cultures tribales, on attache des morceaux de fourrure, de tissu, ou d'autres objets sur une ceinture pour créer des vêtements courts. L'anneau est donc un ur-objet, à la fois un objet concret et un schéma d'expansion radiale.

Alors que le pendentif et l'anneau impliquent déjà une certaine forme de mouvement, la troisième catégorie d'ornement de Semper est encore plus étroitement liée au mouvement. Pour nommer cette dernière, il invente l'expression "ornement de direction" (Richtungsschmuck). Cette forme de parure accentue la direction du mouvement du corps de celui qui la porte, tels que les accessoires des coiffures, les plumes d'un chapeau ou la crête des casques militaires. Bien que l'ornement directionnel ne soit ni rythmique, ni symétrique Semper en fait "le plus spirituel" (die geistigere) de tous les ornements, car il reflète "la grâce, le mouvement, le caractère, et l'expression" de celui qui le porte. ${ }^{28}$

Semper les divise en deux types : l'ornement de direction "flottant" (flatternde) tel que la crête des casques grecs et étrusques; et l'ornement de direction "fixe" (feste, unbewegliche) tel que le serpent sur le front de la divinité égyptienne, ou les casques rigides des Assyriens. ${ }^{29}$ Avec ses oscillations rapides, l'ornement de direction flottant sert de tachymètre, il indique non seulement la direction mais aussi la vitesse du celui qui le porte. D'autre part, alors qu'il ne bouge pas lui-même, le richtungsschmuck fixe, schématise le mouvement du porteur comme une plume écrivant dans l'air. L'ornement directionnel, qu'il soit flottant ou fixe, dessine un deuxième contour virtuel par dessus celui du corps, augmentant la circonférence visible du corps humain ou animal. (Fig.3ab) 
Fig.3a-b
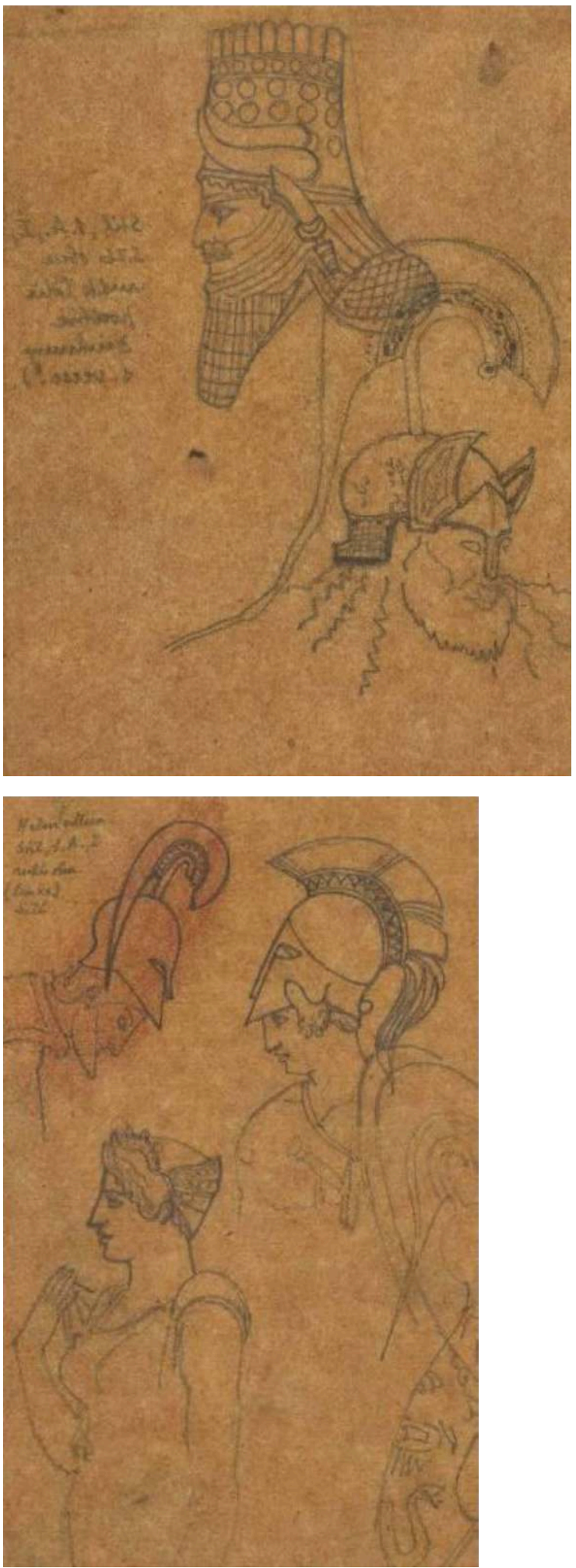

Gottfried Semper, esquisses d'ornements directionnels et annulaires pour la tête-dessins préparatoires (avec instructions manuscrites) pour l'illustration de Der Stil (non datés). Semper Archiv, Institut für Gesichte und Theorie der Architektur (gta) ETH-Zurich. 20-0163-25A (detail) and

20-0163-26A (detail). Semper Archiv, Institut für Gesichte und Theorie der Architektur (gta) ETHZurich. 20-0163-25A (detail) and 20-0163-26A (detail). Photo: gta, ETH, Zurich 
Semper insiste sur l'importance de l'ornement de direction dans l'attirail militaire, notamment dans le cas des bandeaux qui ornent les têtes des cavaliers et de leurs chevaux, comme ceux qui sont représentés sur les bas-reliefs assyriens de Nimrud que l'architecte a vus au British Museum lors de son exil londonien du début des années $1850 .{ }^{30}$ Dans ces bas-reliefs Assyriens, les corps des guerriers et de leurs chevaux sont progressivement remplacés par les signes eidétiques de leurs accessoires. C'est comme si l'ornement directionnel transformait le mouvement lui-même en ornement, en faisant du champ de bataille une arène de lignes, de points, et de motifs fuyants. Ce mouvement projectif devient particulièrement efficace lorsque les contours des chevaux galopant sont doublés ou triplés (comme c'est le cas dans bon nombre de ces bas-reliefs de Nimrud). Le spectateur peut alors reconstituer la trajectoire de mouvement en combinant les traces itératives des ornements (cette perception du mouvement anticipe ostensiblement à la fois la méthode et l'iconographie des expériences chronophotographiques de Muybridge).

Ce n'est pas un hasard si les ornements de direction sont étroitement associés à la hiérarchie visuelle du champ de bataille et à sa nature hautement dynamique. L'une des plus anciennes significations du Kosmos grec renvoyait à la dynamique spatiale de l'organisation militaire. ${ }^{31}$ Avant la guerre contre l'ornement il y a la guerre représentée par le côté cosmique de l'ornement. Essentiellement, les ornements de direction sont les bannières de telles batailles. Ils étaient le signe d'un monde fluctuant, dans lequel les formations régulières, comme celles représentées par l'anneau et le pendentif, ont été progressivement remplacées par une géométrie plus complexes qui a suspendu les modes de hiérarchie visuelle et sociale déjà établies. Alors que les ornements directionnels auraient finalement conduit à la disparition de l'ornementation classique, ils ouvrent la voie à une infinité de nouvelles directions qu'une telle disparition pourrait précipiter.

\section{Accessoires Mobiles (Bewegtes Beiwerk)}

Dans ses notes, Warburg retranscrit un long passage de Semper où il explique que parfois, les étoffes peuvent se ranger dans la catégorie des ornements de direction: “J'ai pu appeler le vêtement à l'état de repos un ornement macrocosmique ; à l'état de mouvement, lorsqu'il flotte en l'air, il faut évidemment le ranger parmi les ornements de direction." ${ }^{32}$ Les étoffes se situent donc entre le mouvement périodique des pendentifs et le mouvement plus linéaire des ornements de direction; elles expriment à la fois l'aspect tellurique et l'aspect cinétique. En raison de leur "légèreté," leur mouvement "reste plus ou moins indépendant de celui de l'individu," mais peut aussi suivre la direction de ceux de l'individu, et leur tenue peut faire ressortir "le sérieux, la solennité, la gravité." Sur une esquisse jamais publiée de son vivant, Semper a reproduit une ménade dansant, d'après un bas-relief néo-attique. On y voit une femme qui marche à grandes enjambées et son vêtement flatteur bat l'air au-dessus d'elle, presque autonome par rapport à son corps. ${ }^{33}$ (Fig.4) 
Fig. 4

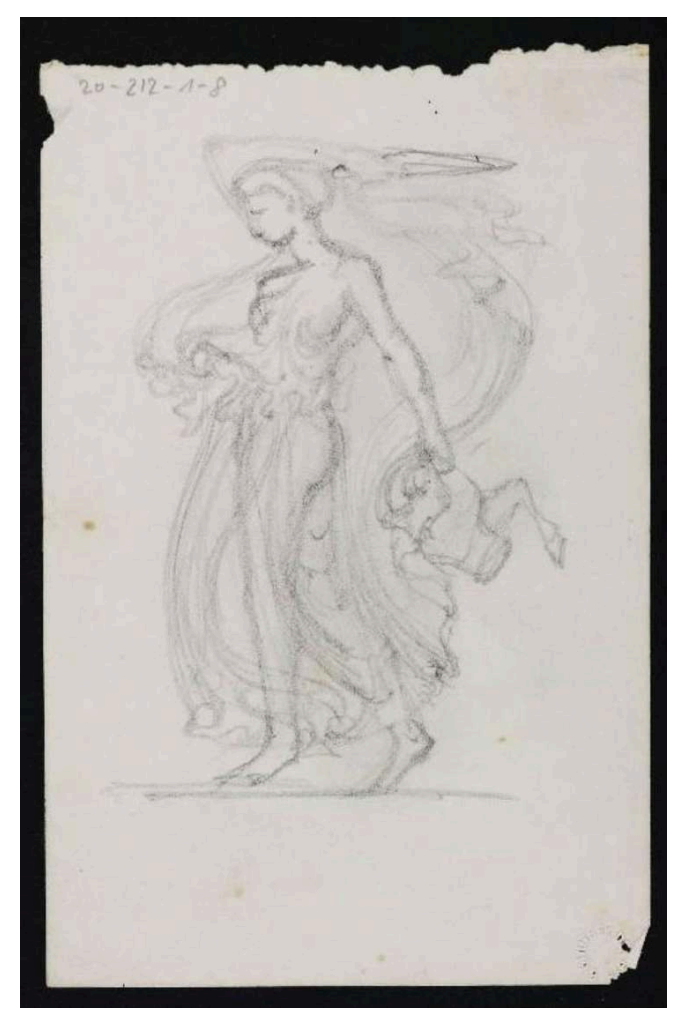

Gottfried Semper, dessin d'une "ménade dansant" d'après un bas-relief néo-attique (fin du 2nd siècle avant JC). Semper Archiv, Institut für Gesichte und Theorie der Architektur (gta) ETH-Zurich. 20-0163-25A (detail) and 20-0163-26A (detail). Semper Archiv, Institut für Gesichte und Theorie der Architektur (gta) ETH-Zurich. 20-0212-8. Photo: gta, ETH, Zurich.

Dans la main gauche, qu'elle a repliée derrière la nuque, la femme tient un couteau qui se trouve dans l'alignement de l'étoffe de son vêtement flottant; il devient un simple vecteur, attirant l'attention sur l'horizontalité du voile, comme si celui-ci était une décoration directionnelle. De sa main droite, la ménade tient le membre postérieur d'une bête abattue. Dans son dessin, Semper donne au cadavre de l'animal le rôle d'objet gravitationnel -tout comme le ferait n'importe quelle poterie- ; il pèse de tout son poids vers la terre, dont l'étoffe flottante nie entièrement la force. L'objet tenu à la main est le contrepoids formel de la draperie qui défie les lois de la pesanteur; la combinaison de ces deux caractéristiques insiste sur la double dimension, tellurique et cinétique, de la parure humaine. Symptomatiquement, quelques décennies plus tard, Warburg inclut une photo du même bas-relief néo-attique dans l'atlas Mnémosyne, parmi les images d'une planche sur le thème du "viol" (Raub) et du sacrifice cérémoniel (Opfer), mais là, le motif du voile donne lieu à une lecture symbolique différente, sans contrepoids épistémologique. ${ }^{34}$

Lorsque Warburg recopie du livre de Semper un paragraphe entier sur l'étoffe en tant qu'élément directionnel, c'est son propre crayon qui devient "l'ornement de direction" guidant les recherches du jeune historien de l'art. En effet, les quatre pages de notes que Warburg a pris sur Der Stil de Semper sont datées de "Florence, Novembre 1888," 35 précisément à la période où il étudiait avec August Schmarsow à l'institut allemand d'histoire de l'art en Italie. Peut-on aller jusqu'à imaginer que ce fut peut-être Schmarsow - qui s'intéressait vivement à l'organisation spatiale et aux principes de composition - qui lui suggéra de lire Semper ?36 
En tout cas, l'intérêt de Warburg pour le mouvement des draperies est né au tout début de sa formation avec Schmarsow. Parmi les notes qu'il prenait pour un cours de Schmarsow sur l'art italien à l'Institut d'art historique de Florence en 1888-1889, il y a une page (datée de décembre 1888) sur laquelle Warburg complète ses notes sur " Les portes du Paradis ", les bas-reliefs de Ghiberti pour le baptistère de Florence, par quelques esquisses fragmentaires de "la bataille contre les Philistins" tirées de "La Vie de David." Ces dessins représentent deux guerriers portant des "étoffes flottantes" (flatternde Gewänder) qui volent dans des "directions opposées" (entgegengesetzten Richtungen). ${ }^{37}$ (Fig.5)

Fig.5

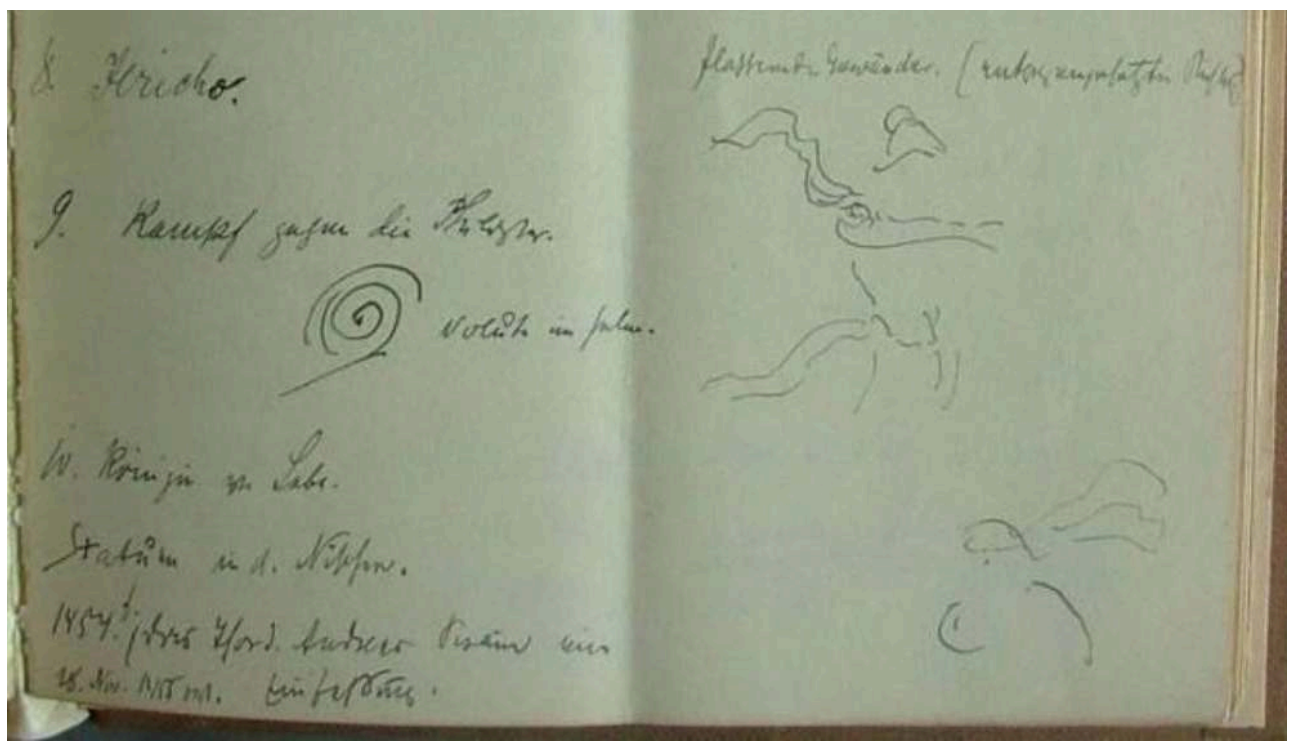

Aby Warburg, notes de cours manuscrites et esquisses: "9. Kampf gegen die Philister.-[F]latternde Gewänder (entgegengesetzen Richtungen). Volute im Helm." Les notes décrivent le bas-relief de Ghiberti sur la vie de David, sur les Portes du Paradis, au batistère de Florence. Aby Warburg, 'Von Nicolo Pisano bis Michelangelo. Vorlesung von Prof. A. Schmarsow gehalten im Kunsthistorischen Institut zu Florenz, W-S 88/89', WIA, III.33.2.8, 67. Photo by the Warburg Institute, London

Dans ses notes, Warburg mentionne aussi la "volute" attachée au casque de l'un des guerriers suggérant ainsi une affinité formelle entre le vêtement flottant et le motif ornemental. Mais quel est le rapport entre la forme libre de l'accessoire mobile et le motif géométrique du casque? Est-ce que les spirales formées par le vêtement pourraient répéter les schémas mentaux de la volute (conformément à l'ornement tel que le théorise Riegl dans son Stilfragen) ? Peut-être, mais pas pour Warburg. Il adhère plutôt à l'origine "dynamique" de l'ornement corporel que développe Semper - surtout en ce qui concerne les étoffes et la coiffure, que Semper appelle "ornements de direction". Ainsi pour eux, les accessoires peints sur les tableaux de la Renaissance ne sont pas le produit d'une stylisation intellectuelle mais d'une transformation matérielle continue. Peut-être que les accessoires représentent ce que Semper aurait appelé "le rudiment" - le fossile minéral du mouvement énergique de la draperie ; mais l'art de la renaissance marque la réanimation de ces ornements auparavant fossilisés par des forces naturelles, culturelles, politiques et économiques. Dans les bas-reliefs de Ghiberti, le mouvement flatteur de l'étoffe est dirigé par les gestes des guerriers. Leurs 
gestes se succèdent dans l'espace agité du champ de bataille, qui finit par dissiper toutes les géométries régulières.

Les quatorze pages de notes que Warburg a prises sur l'essai de Semper sur l'ornement sont plus vieilles d'une année que ses notes sur le cours de Schmarsow; elles sont datées de "Berlin, automne $1890 ",{ }^{38}$ une période pendant laquelle il était profondément plongé dans son travail de thèse, qu'il a défendu en 1891. Dans ses notes manuscrites, qu'il avait inseré dans la version publiée de sa thèse, Warburg cite une phrase de Semper: "l'esthétique de la beauté pure a sa base matérielle dans la dynamique et la statique (Die Aesthetik des Rein-Schönen hat ihre materielle Grundlage in der Dynamik und Statik)." ${ }^{39}$ (Fig.6)

Fig.6

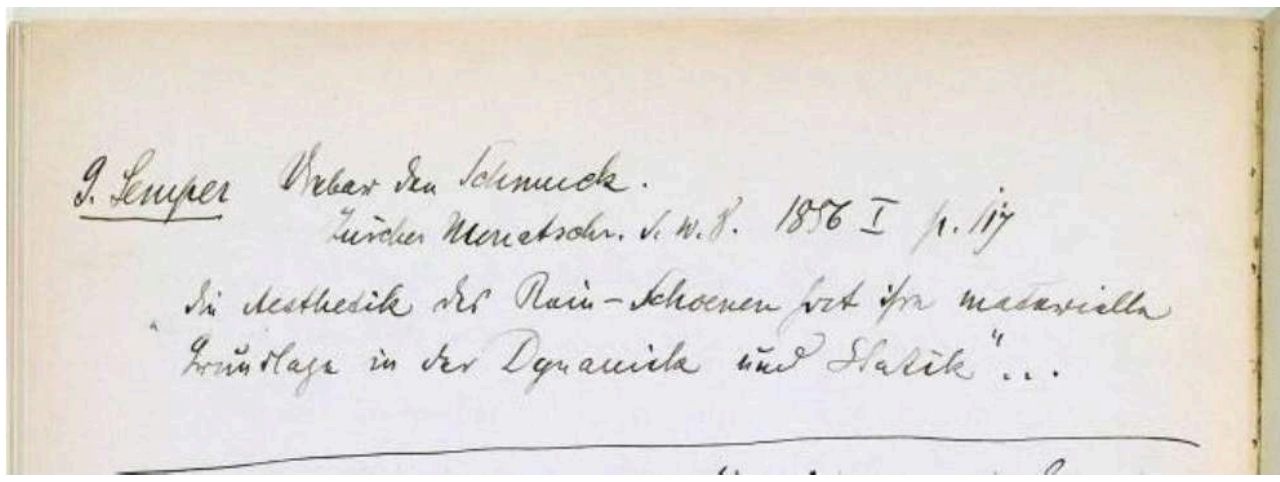

Aby Warburg, ajout manuscrit: "G. Semper, Uber den Schmuck: "Die Aesthetik des Rein-Schönen hat ihre materielle Grundlage in der Dynamik und Statik...." Copie personnelle de sa thèse sur Botticelli, WIA III. 40.1.1.2, p. 10. Photo: Warburg Institute Archive, London

Cette phrase de Semper ne révèle pas seulement l'origine matérielle d'une forme esthétique plus vieille, mais jette aussi les bases d'une nouvelle science esthétique qui tient compte des forces vives, celles en équilibre et celles en mouvement. Bien que la thèse de Warburg soit loin d'avoir pour sujet "la beauté pure," la citation de Semper fait profondément écho à la dynamique picturale des deux tableaux de Botticelli analysés par Warburg dans sa thèse -La Naissance de Vénus et Le Printemps. Ils sont tous deux envahis par une multitude d'accessoires directionnels qui épousent le mouvement du vent. ${ }^{40}$ Même si l'historien de l'art ne fait pas explicitement référence aux trois catégories d'ornement de Semper dans sa thèse, il existe des liens évidents entre ses recherches sur la représentation picturale des accessoires et l'analyse du Schmuck par Semper.

Il n'est pas ici question des bijoux que portent les personnages représentés, tels que les colliers ou les broderies décoratives des Grâces dans Le Printemps, qui ont déjà fait l'objet de l'analyse des historiens de l'art. ${ }^{41}$ Dans l'interprétation de Warburg, chaque tableau fonctionne plutôt comme un ornement à part entière, selon la logique qui structure la définition des trois catégories d'ornement de Semper. Le point de départ de Warburg est la catégorie de l'ornement directionnel de l'étoffe. Comme dans les scènes guerrières des bas-reliefs assyriens, les représentations mythologiques composées par Botticelli se transforment en arènes de courbes tourbillonnantes, tous les personnages représentés étant essentiellement réduits à leurs accessoires mobiles, ils fonctionnent comme des signifiants visuels de l'agitation et de l'inertie. Dans ce cadre, on pourrait entièrement réinterpréter La Naissance de Vénus comme un ornement annulaire pictural 
étant donné que sa chevelure et ses accessoires tournoient autour la Venus marmoréenne qui, pour sa part, constitue un axe statique. A ce titre, la conclusion de Warburg sur l'hésitation de Boticelli entre "la tranquillité mélancolique" et "l'agitation véhémente" ${ }^{{ }_{42}}$ ne transforme-t-elle pas la peinture en un pendentif psychologique, alternant périodiquement entre deux modes d'imagerie diamétralement opposées mais intimement liées?

Il existe des différences fondamentales entre les conceptions sempériennes et warburgiennes du rapport entre l'article d'ornementation et son porteur humain. Dans un aphorisme esthétique écrit en octobre 1890, Warburg revient sur sa lecture de l'essai de 1956 de Semper :

“Dans le cas de l'ornement, la forme d'expression représentée n'a aucune relation organique à celui qui le porte, car [le mouvement à] la surface [agitée] ne représente pas le résultat naturel de la puissance instantanée ni la volonté du porteur." ${ }^{43}$

Contrairement à Semper, pour Warburg, le rapport entre l'ornement et les émotions subjectives de son porteur n'est pas transparent. Warburg insiste sur l'indépendance de l'ornement inorganique par rapport au moi organique, comme si l'artefact décoratif possédait une volonté propre et s'exprimait de lui-même.

Une autonomie virtuelle est centrale pour la pensée de Warburg. Cela lui permet de reconceptualiser la pensée de Semper sur la parure (Schmuck) en "accessoire mobile" (Bewegtes Beiwerk), similaire à la chevelure tourbillonnante et à l'étoffe balayée par le vent dans La naissance de Vénus de Botticelli. Le point commun entre la parure et l'accessoire est leur qualité d'élément "adjoints" par l'ornementation : le processus par lequel quelque chose en plus est ajouté à ce que le corps organique a à offrir à la représentation, précisément grâce à un élément inorganique. Dans plusieurs de ses brouillons de thèse, Warburg caractérise ces mêmes accessoires $d$ ' "additifs dynamiques et extensifs" (Zusätze) lorsqu'ils apparaissent en peinture ou en littérature. 44

Comme l'a montré Warburg dans ses analyses minutieuses des accessoires décrits dans les textes de l'Antiquité et de la Renaissance, les ornements prolifèrent par adjonctions et embellissement continu. Les artistes et les écrivains de la Renaissance ont tendance à "accessoiriser" l'accessoire en ajoutant une courbe, un gribouillis, une fioritura de plus à l'étoffe déjà ornementée de l'antiquité. Cette méthode d'auto-engendrement constitue la loi suprême de l'accessoire, mais elle se situe en dehors des chemins de formations établis par Semper. Le Beiwerk de Warburg semble n'obéir à aucune autorité et évolue même "contre le cours naturel du monde." ${ }^{35}$ L'accessoire mobile n'obéit qu'à un seul principe, qui est incompatible avec la vision sempérienne d'un univers ordonné, c'est le principe du changement imprévisible. Alors qu'il écrit une trentaine d'années après l'architecte, Warburg réalise peut-être que le monde et ses formes d'art sont devenues de plus en plus ornementales, mais pas dans le sens des principes cosmiques de Semper. Les appendices décoratifs ne réalisent plus l'ordre de monde, mais contribuent à un désordre généralisé qui suscite l'inquiétude cosmologique de Warburg. Pour reprendre les termes de l'épigramme des Prolegomena l'homme "satisfait ses instincts cosmogoniques" en créant des artefacts décoratifs, mais il ne cherche pas à démystifier le monde en cherchant dans leur forme la réflexion de principes et de lois scientifiques. $\mathrm{Au}$ contraire, par une abondance de moyens techniques, il s'efforce de reproduire et de renforcer la "merveille" - le mystère radical de la nature, qui avait initialement piqué sa curiosité. 


\section{La survivance de la parure}

Alors que le nom de Semper n'est pas mentionné dans la publication originale de la thèse de Warburg, il apparaît quelques années plus tard, dans une note de bas de page de son essai de 1895, "Costumi Teatrali". Il décrit "les étoffes flottants, les volants, les draperies" utilisés pour ces performances et précise qu'ils étaient conçus pour être vus en mouvement et "de profil" comme "en cortège," dans sa note il ajoute que "Gottfried Semper a inventé le mot juste pour ce genre de décoration, Richtungscmuck [sic]." ${ }^{46}$ Bien que marginale, cette note souligne une transition importante: pour Semper, l'ornement de direction structure surtout l'espace militaire au moments même de l'action sur le champ de bataille, mais pour Warburg, il fait partie de "l'espace à jouer" (Spielraum) de la pompe et du spectacle - il appartient plus au domaine de l'imitation de l'action qu'à celui de l'action elle-même. L'ornement garantit la survivance de la vie sociale et culturelle, mais uniquement sous la forme de vestige, en tant que rudiment des fonctions vitales précédentes qui se sont atrophiées en appendices décoratifs flottants et indépendants. C'est là l'évolution la plus générale de l'ornement dans la culture mondiale de la fin du XIXe siècle; la réception historiographique des théories de Semper sur l'ornement indique leur survivance sous une forme rudimentaire mais festive.

Car vraisemblablement, Warburg n'est pas le seul lecteur méconnu de Semper. Bon nombre d'ornementologistes ont recopié et réutilisé ses catégories de la parure. Je n'en mentionnerai que deux d'entre eux, dont les titres des œuvres figurent dans les notes bibliographiques de Warburg sur Schmuck dans les Zettelkästen (les boîtes en carton où il conservait ses recherches et ses "notes scientifiques"). Le premier de ces auteurs est J. Matthias, un lecteur en arts appliqués, dont l'étude, publiée en 1870, s'intitule La Parure Humaine (Der menschliche Schmuck). ${ }^{47}$ Le but du livre, comme l'annonce le soustitre est de "contribuer à l'éducation du goût des milieux domestiques et commerciaux." Il s'agit essentiellement d'un guide contre la menace imminente d'un schmuck qui, s'il n'est pas règlementé, devient signe de mauvais goût (schlechter Geschmack). "Il a pour but d'éveiller un sens de la beauté chez les ménagères," écrit Warburg de Matthias. Cependant, plus loin, il critique son attitude intellectuelle frauduleuse : "il se fonde sur Semper sans le citer." ${ }^{48}$

En effet, Matthias classifie les formes de parure féminine selon les trois catégories structurelles de Semper - ornement pendentif, annulaire et directionnel - sans pour autant citer son nom. Le nom de Semper ainsi que tout son programme cosmologique disparaît complètement du texte de Matthias. Peut-être le plagiat éhonté de Semper est-il une nouvelle instance du phénomène de recopiage mécanique de l'ornement sans le moindre égard pour la source originelle (et dans ce cas ce phénomène irait jusqu'à s'appliquer à la théorie de l'ornement elle-même) ? L'essai de Semper a finit par être victime d'un phénomène d'ornementation se reproduisant mécaniquement d'une manière irréfléchie contre lequel il résistait par son travail, ainsi que Warburg après lui.

La seule contribution originale de Matthias est un ensemble d'illustrations de bijoux et de vêtements reproduits d'après des œuvres de la Grèce antique exposés au musée archéologique de Berlin, sans mentionner les dates et l'origine des objets. Alors qu'il se réfère abondamment à l'habillement moderne, il n'insère aucune illustration de 
vêtements ou de parures contemporains. Comme le mouvement d'un pendule, l'histoire et la pratique de "la parure humaine" semble osciller entre les deux définitions sempériennes du cosmos: entre la faculté cosmique de l'archéologie telle qu'elle se dégage des illustrations de l'antiquité de Matthias, et l'ère de l'anthropologie culturelle - une science ravivée par les innombrables objets décoratifs importés des colonies que l'on amasse dans les musées d'ethnographies et qui connaissent la célébrité grâce à un nombre important de publications relatives à la parure du corps à l'échelle mondiale. ${ }^{49}$

Le deuxième essai sur l'ornementation mentionné dans les notes de Warburg se base exclusivement sur ce nouveau monde découvert par l'anthropologie culturelle : C'est $L a$ Parure du Peuple (Der Schmuck des Menschen, Berlin, 1990) d'Emil Selenka, évolutionniste, ethnographe amateur, et collègue de Karl Gottfried Semper, le neveu morphologiste de l'architecte. ${ }^{50}$ (Fig.7a-b)

Fig.7a

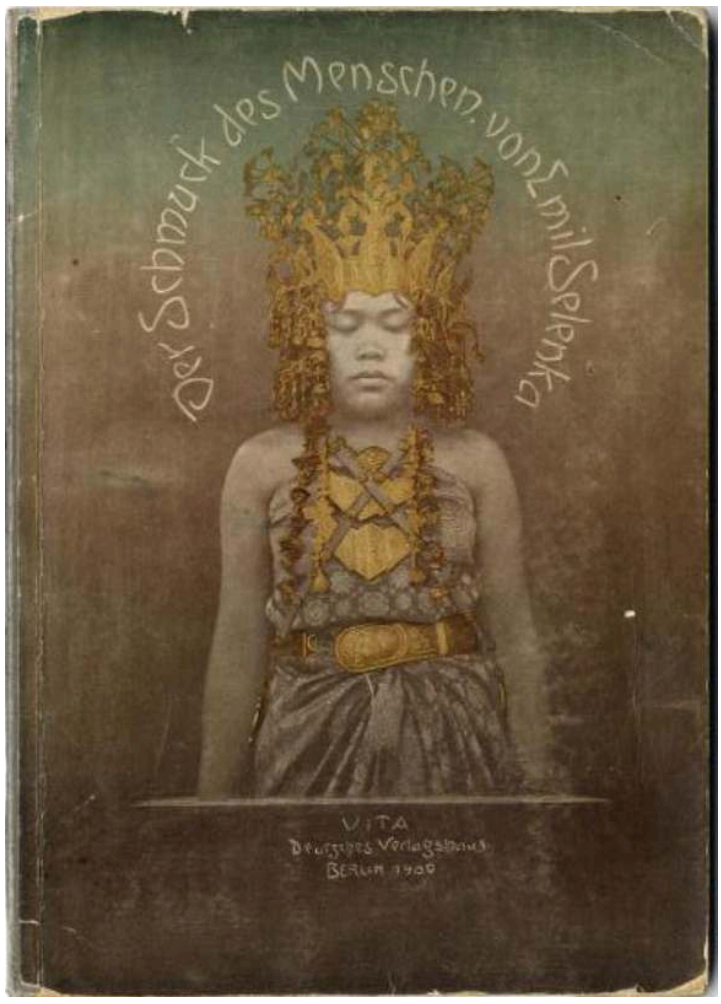

Emil Selenka, Der Schmuck des Menschen (Berlin: VITA Deutsches Verlagshaus, 1900), première de couverture. 
Fig. $7 \mathrm{~b}$

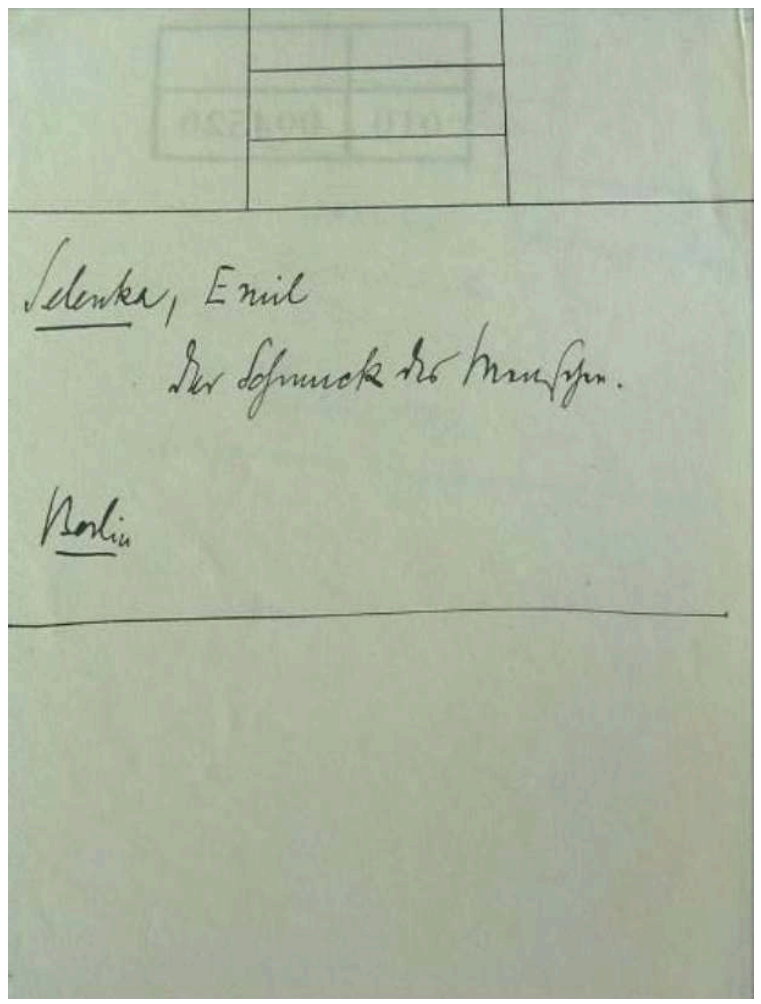

Aby Warburg, note bibliographique: "Emil Selenka, Der Schmuck des Menschen, Berlin 1900" Warburg Institute Archive, Zettelkasten in Zettelkasten titled Gerät-Tracht, WIA ZK 29A 010/004526. Photo: Warburg Institute Archive, London

Avec sa femme, il voyage beaucoup en Asie du Sud-Est, dans les îles allemandes, des Philippines à Sumatra. Ils collectent des fossiles et d'autres spécimens pour leur recherche scientifique sur l'évolution des espèces de primates et, dans le même temps, Selenka accumule un grand nombre d'informations sur les coutumes et les différents motifs de parure des peuples de ces territoires. ${ }^{51}$

Selenka utilise les trois catégories structurelles de Semper pour présenter la parure comme un "langage universel des images" (Bildersprache). Contrairement à Matthias, il rend hommage à l'architecte, même si son opinion diverge parfois de la sienne et l'amène à apporter ses propres embellissements au schéma ornemental initial, auquel il ajoute trois catégories de plus. L'évolutionniste applique les catégories sempériennes strictement aux cultures non-occidentales, car ces peuples "vivent tout proche de la nature" et pour cette raison, leur sens instinctif de l'ornement n'est pas altéré par des goûts préalables. Dans les illustrations abondantes de l'ouvrage, les pendentifs antiques de Semper se transforment en longues tresses comme celles des fakirs indiens; les propriétés proportionnelles des anneaux sont étendues aux bijoux métalliques que portent les femmes tamil (Inde) aux chevilles et aux orteils; et pour finir, les avantages militaires conférés par l'ornement de direction se retrouve dans les coiffures en plumes des Apaches et des guerriers malaisiens. Pourtant, ce qui apparaît comme une expansion phénoménale de la culture de l'ornement, ne présage en fait de rien d'autre que de son déclin imminent. Tout en louant la sensibilité ornementale des peuples non occidentaux au détriment des occidentaux dont il omet de parler dans son étude, Selenka cherche à s'assurer que ce qu'il appelle "l'ornement du peuple" est en fin de 
compte l'ornement de ces peuples qui habitent la périphérie exotique du monde. Ce n'est peut-être pas un hasard si la mariée apprêtée qui orne la couverture du livre de Selenka porte au-dessus de sa coiffure de cérémonie, le titre L'ornement $d u$ Peuple, comme une décoration supplémentaire ("Ansatzschmuck" dans les mots de Selenka). Comme un accessoire amovible, l'auréole lumineuse des lettres préfigure l'éclipse de l'ornement annoncée quelques années plus tard par un autre lecteur de Semper, l'architecte Adolf Loos. $^{52}$

\section{L'ornement comme définition épistémologique} plus de ses notes sur la langue indigène, ses carnets de poche sont pleins de notes et de dessins représentant des symboles ornementaux. Warburg a dû copier ces motifs ou ces objets décorés sur des livres qu'il a trouvés dans les bibliothèques américaines, à moins qu'il ne les ait dessinés sur place pendant son séjour chez les Pueblos. ${ }^{54}$ Warburg alterne entre plusieurs termes pour désigner les objets pueblos - Schmuck, Gerät, et Tracht (parure, instrument et costume) - et non pas Beiwerk (accessoire) le terme qu'il utilise dans sa thèse sur Botticelli. Beaucoup des ornements que portent les indigènes entrent dans les catégories de Semper, tels que les pendentifs et les ornements annulaires. Warburg reproduit en détail les différentes formes de coiffures cérémonielles et les masques rituels, dont beaucoup ont une forme circulaire. (Fig.8) 
Fig.8

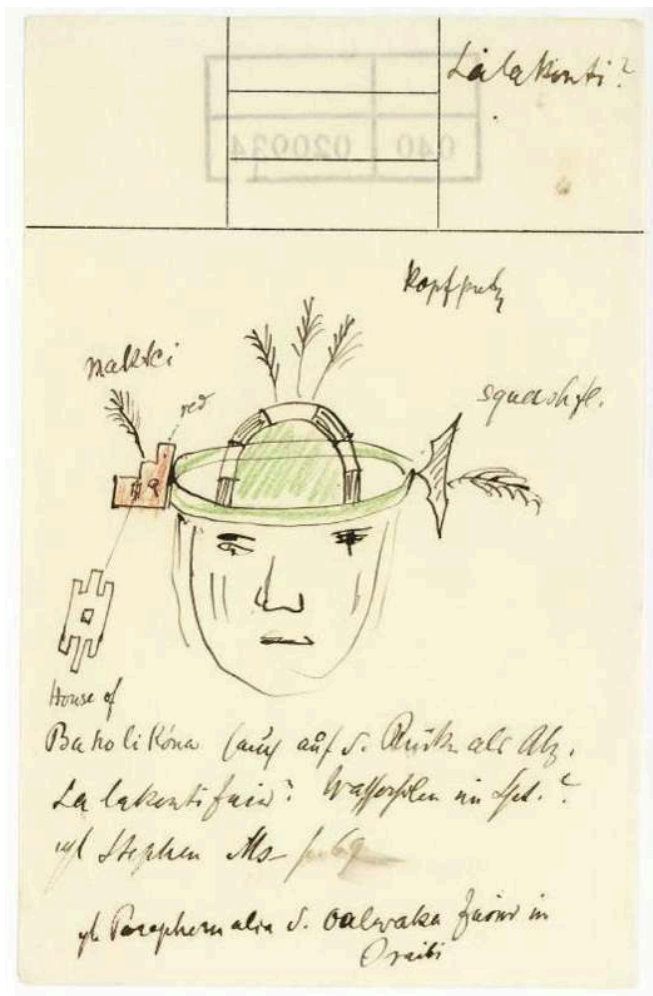

Aby Warburg, esquisse d'un casque moki (Kopfschmuck) en forme d'ornement annulaire portant l'inscription "maison de Baholi Kóna," Warburg Institute Archive, Zettelkasten Americana, WIA, ZK 040/020934. Photo: Warburg Institute Archive, London.

47 Même le dieu de la pluie serpent porte une plume sur la tête, "l'ornement de direction" par excellence selon Semper.

D’une manière analogue aux ornements de la théorie "Post-Newtonienne" de Semper, de nombreux motifs ornementaux décorant les poteries des Pueblo sont des symboles de forces cosmiques naturelles. En effet, si l'ornement sempérien représente le monde, l'ornement Pueblo l'incarne. Par exemple, dans les dessins de Warburg, la simple spirale dénote le vent, le tourbillon masculin en particulier. La double spirale et les lignes en zigzag semblables à un escalier, sont des symboles cosmiques largement répandus sur les poteries pueblos. ${ }^{55}$ (Fig.9) 
Fig.9

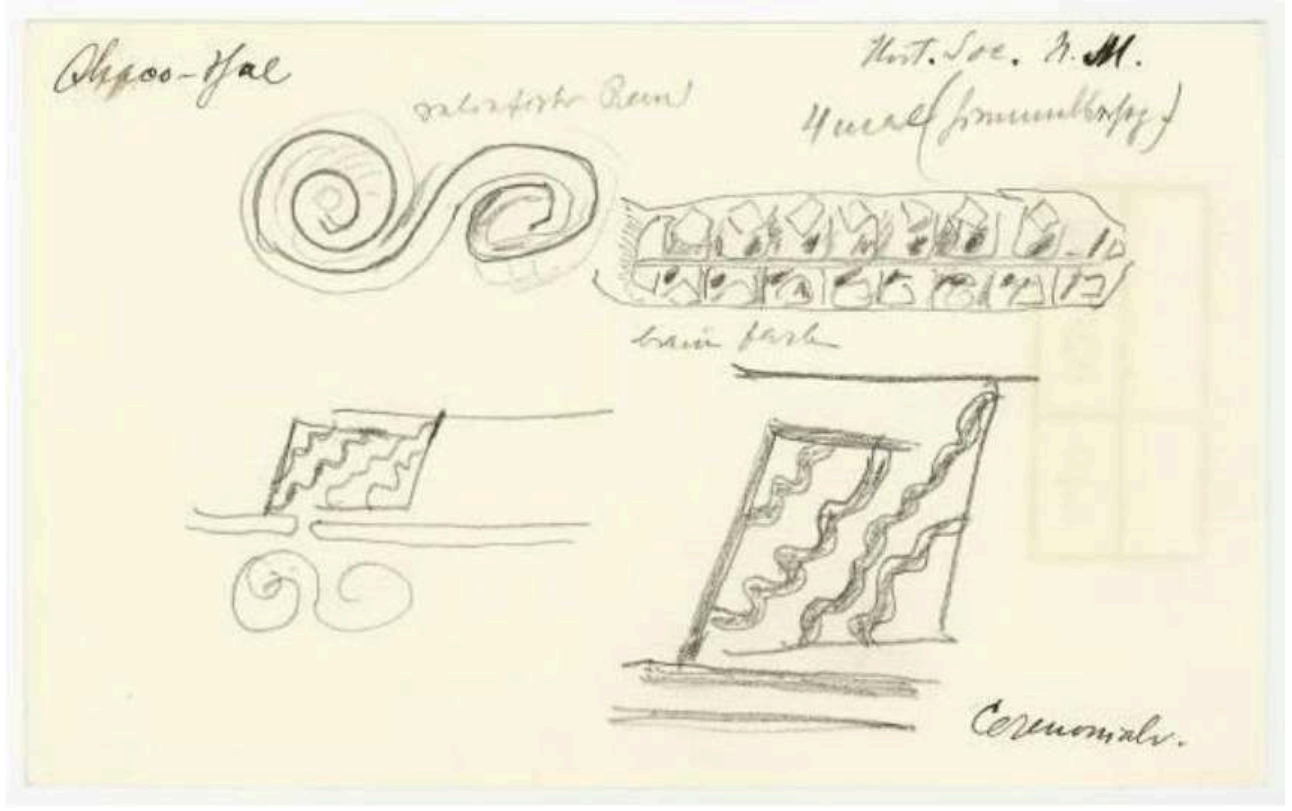

Aby Warburg, esquisse d'un ornement pueblo en forme de spirale, Zettelkasten Americana, WIA, ZK, 040/020762. Photo: Warburg Institute Archive, London

Les notes de Warburg contiennent également plusieurs dessins miniatures qui représentent les différents symboles de la foudre: un serpent dont la tête est une flèche, un escalier qui fait partie de la maison du monde, les nuages, et un dessin en forme de peigne qui représente la pluie. En bas de la page, tous les motifs précédents sont combinés en un seul, le célèbre symbole pueblo de "la maison du monde." ${ }^{56} \mathrm{Chez}$ les Pueblos, les forces physiques ne sont pas seulement représentées par des schémas géométriques mais aussi par des objets concrets ou des corps d'animaux. Dans l'étude ethnographique d'Adolph Bandelier des symboles tehua et zuni, même l'arc en ciel, représenté par une bande curviligne colorée, est doté d'une petite tête et de deux pieds à ses extrémités.$^{57}$ L'animal est le Kosmos.

Parmi tous ces symboles, Warburg s'intéresse tout particulièrement à celui de "la maison du monde". Ce motif cosmologique montre le firmament qui se referme sur les nuages. De nombreuses versions de ce symbole, dont certaines en couleur, figurent dans les croquis de Warburg. ${ }^{58}$ Dans certains d'entre eux, la maison du monde est représentée par des contours angulaires, et dans d'autres par un motif semi-circulaire avec des volutes, flanqué d'animaux totémiques de chaque côté. Selon la terminologie sempérienne, la ligne semi-circulaire qui entoure le monde serait un ornement annulaire "périphérique." (Fig.10) 


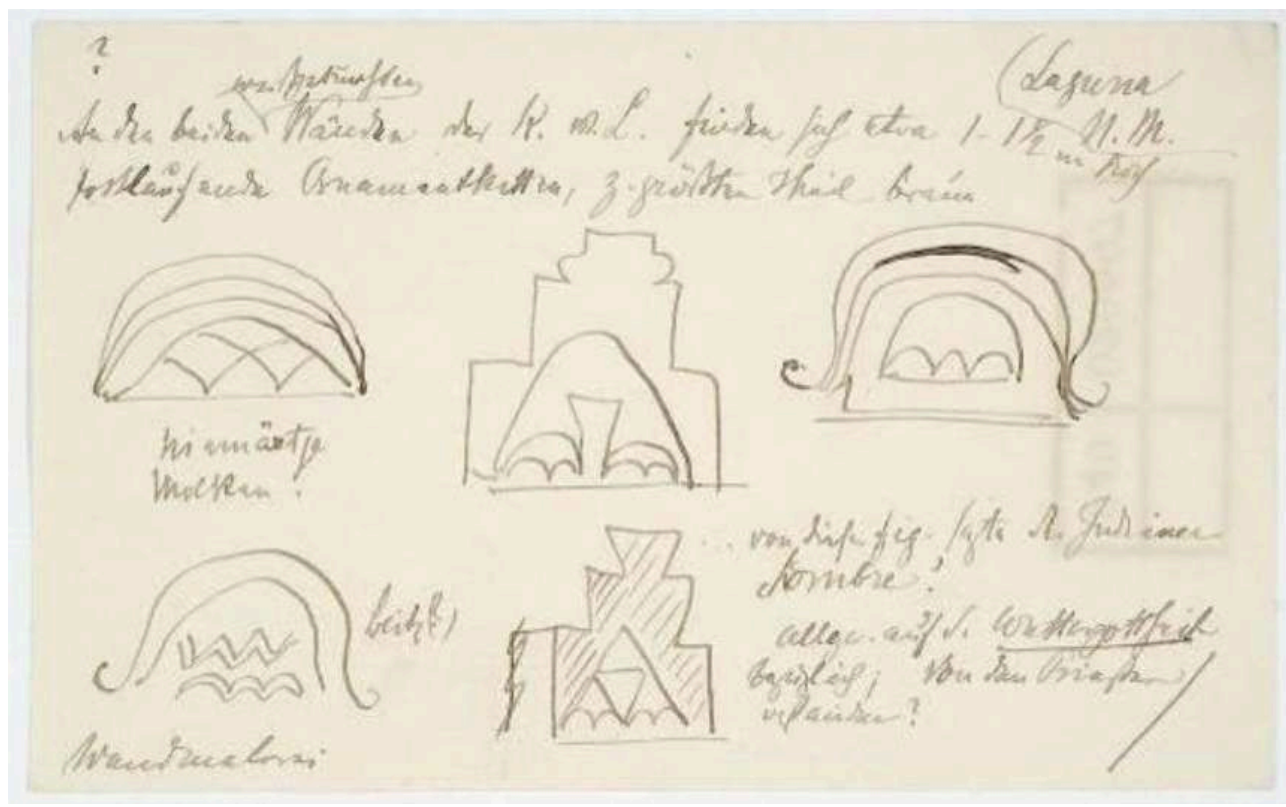

Aby Warburg, esquisse des symboles Pueblo de "I'ornement marche" et de "la maison monde," Zettelkasten Americana, WIA, ZK, 040/020697. Photo: Warburg Institute Archive, London.

51 Mais pour Warburg, cette même ligne pourrait également représenter l'idée de "Umfangsbestimmung" (la définition d'une périphérie conceptuelle ou la circonférence d'une idée), qui vient ici souligner la définition spéculative du Kosmos en fonction de la culture ou de la mentalité..$^{59}$ L'Umfangsbestimmung définit par l'arc en ciel des Pueblos ou le symbole de la maison du monde, est un enclos semi-circulaire qui peut s'étendre à l'infini. Personne ne peut dessiner de limites sur l'espace du ciel, mais il est peut-être possible de créer un ornement à partir de cette frontière impossible. "La maison du monde" des Pueblos montre justement comment un symbole cosmique peut devenir une allégorie en exhibant son incapacité à circonscrire son sens à l'intérieur des limites d'un contour..$^{60} C^{\prime}$ est peut-être grâce à cet ornement pueblo que Warburg est parvenu au plus près d'une compréhension sempérienne de l'ornement, en tant qu'idéogramme symbolique de l'ordre cosmique. Cependant avec Warburg, cet ordre devient de plus en plus allégorique, conformément à son projet de redécouverte de "la genèse de l'ornementation," sans fin et sans espoir de conclusion. ${ }^{61}$

Même si de nombreux ornements cérémoniaux des fêtes pueblos ressemblent aux anneaux, pendentifs, et autres ornements de direction de Semper, les recherches de Warburg sur l'ornement pueblo renforcent l'idée de capacité idéographique de l'ornement qui n'est que timidement suggérée dans sa thèse de doctorat. Pour Warburg, les trois catégories sempériennes fonctionnent comme des idéogrammes du mouvement, si non physique, du moins conceptuel; quoiqu'ils prennent bien leur source dans des objets réels, ils expriment des modes de rapports et d'être dans le monde.

On pourrait peut-être trouver le germe de la pensée de Warburg dans son interprétation de Sartor Resartus, un livre de Carlyle qui décrit le monde naturel et humain comme la superposition de plusieurs épaisseurs de vêtements. ${ }^{62} \mathrm{En}$ interprétant les catégories d'ornement en fonction de Carlyle, Warburg renverse le modèle de Semper. Pour Semper, Schmuck représente le vestige matériel, "le rudiment" 
des transformations socio-culturelles et artistiques, mais pour Warburg, c'est son origine secrète, le germe invisible interdisant une définition ultérieure de l'art. La différence réside dans l'orientation conceptuelle (Orientierung) aussi bien que dans la direction (Richtung) de la signification historique et épistémologique de la parure.

Sur le dessin d'un pendule en mouvement, qui accompagne ses aphorismes esthétiques rédigés pendant son voyage américain, Warburg a écrit le mot "Umfangsbest" [? illisible]. ${ }^{63}$ (Fig.11)

Fig. 11

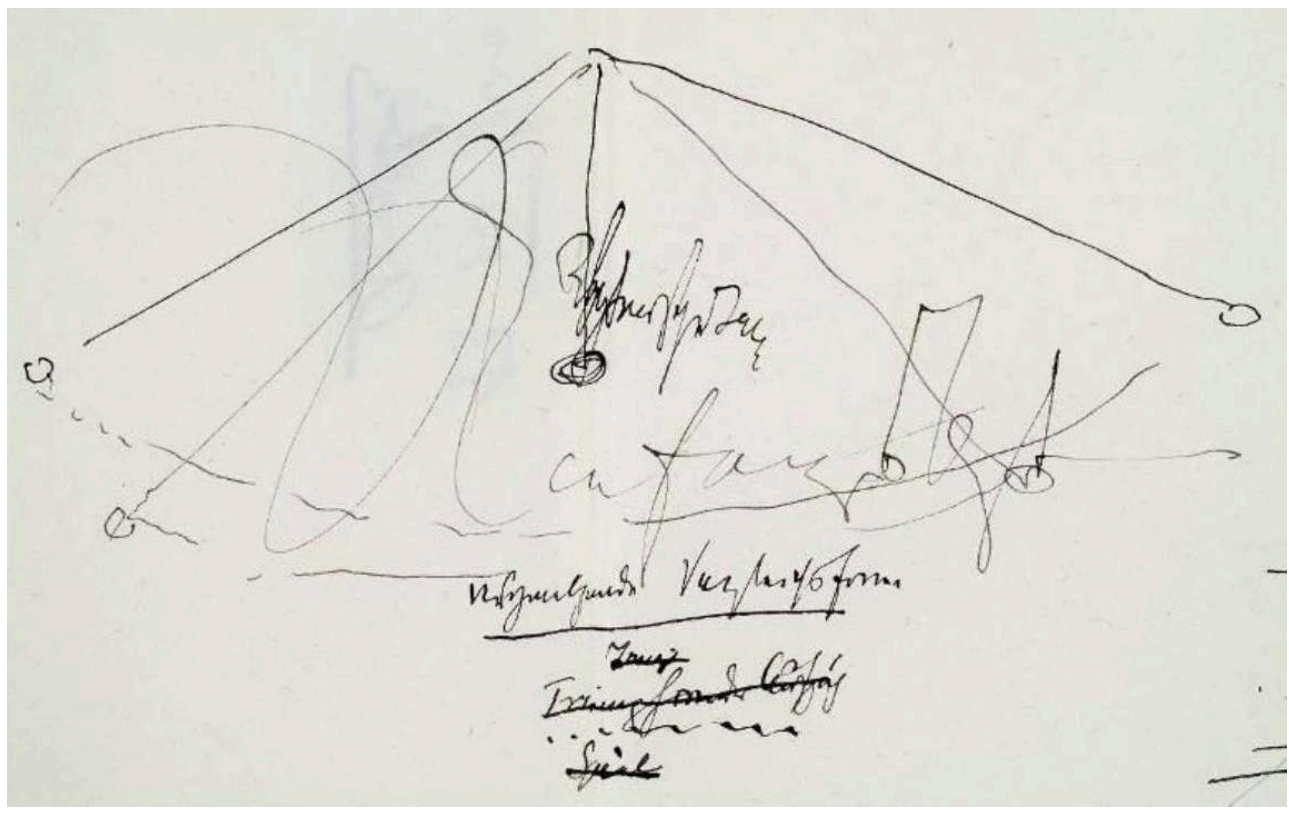

Aby Warburg, esquisse de pendule représentant I'Umfangsbestimmung. Warburg Institute Archive, Zettelkasten Ae.[sthetik]-Aphorismen, Nr. 399. WIA, ZK [not numbered]. Photo: Warburg Institute Archive, London.

Cette page recouverte de pensées sur la fonction symbolique de l'ornement est précieuse car elle est ne se contente pas de juxtaposer l'image du pendule au texte, mais y superpose aussi graphiquement la notion d'Umfangsbestimmung. Cette coïncidence renforce l'idée que sa vision de l'Umfangsbestimmung est elle-même dynamique et oscillante. De la même manière, le motif de la "maison du monde" des Pueblos démarque un horizon conceptuel simultanément ouvert et fermé. Par conséquent il ne parvient pas à délimiter précisément un espace: chaque Umfangsbestimmung reste essentiellement indéfini (unbestimmt). Comme la circonférence idéationnelle définie par le mouvement d'un pendentif, toutes les définitions épistémologiques oscillent continuellement.

Il est cependant remarquable de voir, à travers l'étude de Warburg, comment certains des ces idéogrammes épistémologiques indéfinissables se matérialisent en artefacts tridimensionnels et deviennent des objets concrets. Tel est le cas lorsque, par exemple, le dessin géométrique unidimensionnel de la maison du monde se retrouve dans le motif et la forme des coiffures cérémonielles que portent les danseurs pueblos (et Warburg lui-même, comme l'atteste une de ses photos de voyage). Ces sujets lourdement couronnés semblent porter leurs modèles cosmologiques sur la tête. Ils poussent encore plus loin la communication avec l'univers en greffant à leur coiffure 
des antennes - des appendices faits en plumes (bahos), qui signalent un rapport privilégié avec le ciel, à la manière de "l'ornement de direction" (Richtungsschmuck) de Semper.

57 Au cours des danses rituelles, ces mêmes appendices flottants s'organisent en schémas rectilignes, créant ainsi un espace architectural - un champ hautement réglementé mais toujours animé par l'intensité des corps qui bougent en rythme. Peut-être qu'alors, au milieu de cette rupture extatique, entre frénésie et régulation, l'ordre cosmique de l'ornement tel qu'envisagé par Semper et abandonné par Warburg, pourrait être rétroactivement restauré. L'espace d'un instant, l'ornement pueblo peut tenter de regagner "la pesanteur" que Semper avait conférée à tous les objets terrestres, mais qui avait disparu sous la pression de l'accessoire "maniériste." Malheureusement, un tel équilibre cosmique ne peut durer bien longtemps.

Le nouvel effondrement du cosmos est introduit par un autre Schmuck qui fait désormais parti du costume (Tracht) métropolitain contemporain et de ses extensions technologiques infinies. Je veux ici parler du chapeau "haut-de-forme" (Zylinder) de l'oncle Sam descendant Market street à San Francisco parmi les câbles électriques, sur une photo prise par Warburg. ${ }^{64}$ (Fig.12)

Fig. 12

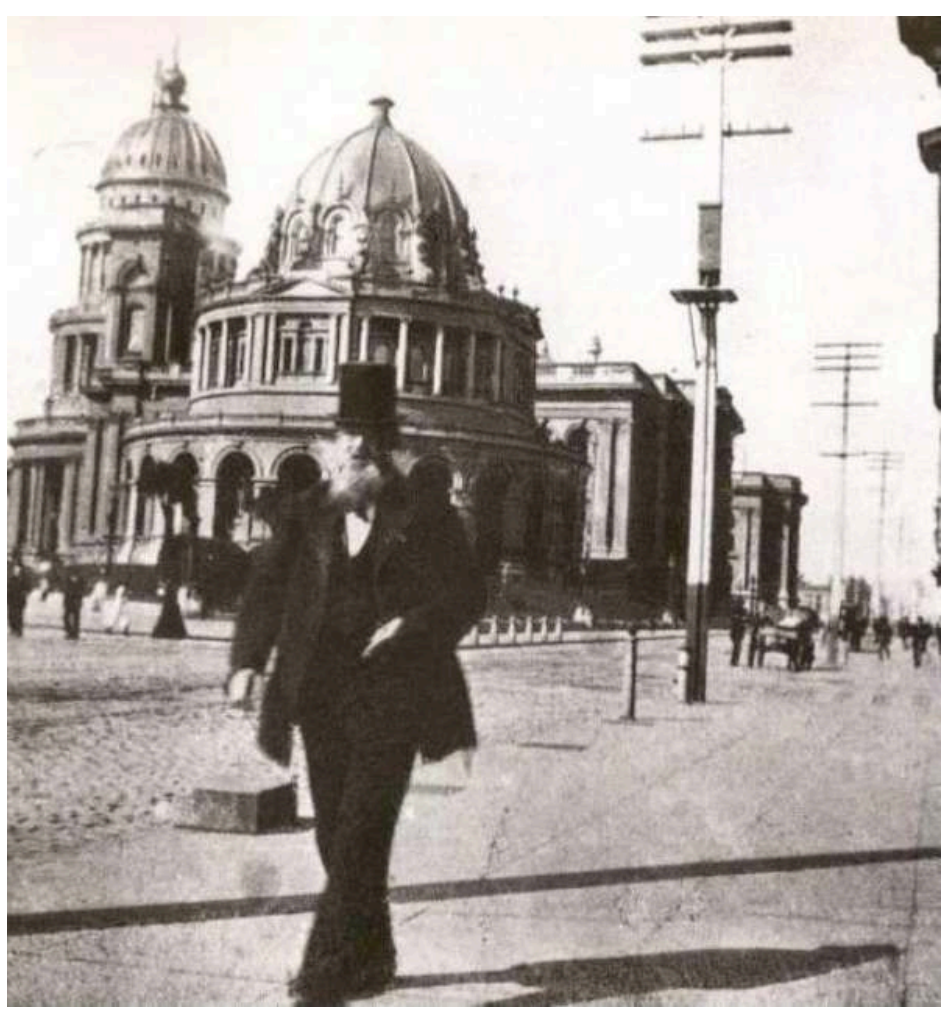

Aby Warburg, photographie de I"Oncle Sam,' San Francisco, Février 1896. Photo: Warburg Institute Archive, London.

Le chapeau haut-de-forme est une autre forme d'ornement de direction sempérien, qui augmente non seulement la taille mais aussi la circonférence idéationnelle de l'homme moderne. Son importance cosmique est renforcée par la rotonde de la Renaissance qui se profile à l'arrière-plan de la photo. Ce bâtiment à la façade travaillée amplifie la forme du chapeau et transforme l'oncle Sam en Américain néo-renaissant. Grace à une 
coïncidence ingénieuse, l'axe central du chapeau de l'individu est aligné avec l'axe de symétrie de la coupole semi-sphérique de la rotonde. Mais dans l'instantané de Warburg, l'axe du chapeau est visiblement faussé. Ce décalage annonce l'échec - peutêtre irrémédiable - de la pesanteur initiale et de l'équilibre classique du cadre architectural de la Renaissance. Contrairement aux esquisses que Warburg a dessinées d'après les principes morphologiques de Semper, le système géométrique sur lequel toutes les analogies cosmiques entre les objets naturels et artificiels étaient fondées, n'est plus rectangulaire mais plutôt oblique et ce biais est l'effet secondaire d'une forme de pesanteur imposée de l'extérieur.

60 Comme le déplore Selenka à la fin de son Der Schmuck des Menschen, la nouvelle génération s'est "débarrassée" de l'ornement qui n'est plus visible que dans les musées d'ethnographie. Bien qu'il soit lui-même un scientifique, l'évolutionniste blâme les sciences naturelles (Naturwissenshaften) d'avoir fait disparaître la parure et "l'augmentation de la circulation" des hommes et des biens qu'elle a produite dans la vie métropolitaine. ${ }^{65}$ Le sens désorientant de la circulation jette les hommes les uns contre les autres dans une multitude déformée d'images contradictoires, il n'y a aucun rythme distinct, aucun axe de symétrie à partir desquels les contours individualisant de la parure pourraient se développer. En d'autres termes, il ne peut y avoir aucun ornement dans un monde moderne parce qu'il n'y a pas de distance entre les corps, les objets et les environnements - il manque cet espace de réflexion et de l'analogie qui rend possible l'existence de l'ornementation. Cette conclusion séduisante fait écho à la fin glorieusement pessimiste du cours de Warburg, Le Rituel du Serpent (présenté en 1923) : où "le meurtre" du Denkraum (l'espace de réflexion) et l'élimination de la distance par la technologie, signale la dissolution du cosmos et (implicitement) la fin de la mentalité analogique qui avait donné naissance à la pratique de l'ornementation à l'échelle mondiale. ${ }^{66}$ Pourtant, c'est précisément de cet état universel apparemment chaotique qu'un nouvel ordre d'ornementation pourrait émerger.

61 De la portée restreinte des ornements pendentifs jusqu'aux extensions infinies des coiffures directionnelles et de leurs arrangements linéaires lors de célébrations festives, l'ornementation du corps est passée de deux à trois dimensions, du Spielraum de la vie quotidienne au Denkraum épistémologique de l'esthétique moniste de Warburg. Le chapeau cylindrique de l'oncle Sam et les coiffures maison du monde des danseurs pueblos signalent tous deux l'importance capitale de la parure dans la culture de la fin du XIXe siècle. La théorie de l'ornement "cosmique" de Semper et son recyclage par Warburg, Selenka, et bien d'autres, montre qu'en l'absence même de pratiques artistiques, l'ornementation reste toujours implicitement au cœur de l'historiographie moderniste, elle continue à générer des représentations textuelles qui tournent comme des satellites autour de la soi-disant éclipse de l'ornement. L'ornement ne représente pas simplement un objet mais aussi la méthode même de la recherche warburgienne: elle divise, bifurque et oscille, pour démontrer que le Verzierungstrieb - que Semper appellerait la pulsion de décorer - partage une racine commune avec la pulsion de savoir 


\section{NOTES}

1. "Umgeben von einer Welt voller Wunder und Kräfte, deren Gesetz der Mensch ahnt, das er fassen möchte, aber nimmer enträthselt, das nur in einzelnen abgerissenen Akkorden zu ihm dringt und sein Gemüth in stets unbefriedigter Spannung erhält, zaubert er sich die fehlende Vollkommenheit im Spiel hervor, bildet er sich eine Welt im Kleinen, worin das kosmische Gesetz in engster Beschränktheit, aber in sich selbst abgeschlossen, und in dieser Beziehung vollkommen hervortritt; in diesem Spiel befriedigt er seinen kosmogonischen Instinkt." Gottfried Semper, Der Stil in den technischen und tektonischen Künsten; oder, Praktische Aesthetik: Ein Handbuch für Techniker, Künstler und Kunstfreunde, Band 1 (Frankfurt am Main: Verlag für Kunst \& Wiseenschaft, 1860), p. xxi.

2. Gottfried Semper, "Über die formelle Gesetzmässigkeit des Schmuckes und dessen Bedeutung als Kunstsymbol," Monatsschrift des wissenschaftlichen Vereins in Zürich 1 (1856): 101-130. La conférence a été reproduite dans Gottfried Semper, Kleine Schriften, ed. Hans and Manfred Semper (Berlin and Stuttgart: W. Spemann, 1884), pp. 304-343. Une traduction française de la première partie de la conférence, par le théoricien esthétique et politicien Paul Challemel-Lacour (qui a fait la connaissance de Semper à Zurich à la fin des années 1850) a paru dans une revue littéraire en 1865 : Gottfried Semper, “De l'ornementation et du style; de leur signification symbolique dans l'art (première partie)" Revue des Cours Littéraires 2:32, 8 juillet, 1865. Toutes les citations de la conférence se réfèrent à la publication intitiale de 1856 en allemand, l'édition à laquelle Warburg se réfère dans ses notes.

3. Pour les notes de Warburg sur la conférence de Semper de 1856, voir Aesthetik, Warburg Institute Archive (hereafter cited as WIA), Zettelkästen (ZK) 041/021140-154; pour ses notes sur Style, WIA ZK 041/021155-158.

4. Semper, "Über die formelle Gesetzmässigkeit des Schmuckes" (voir note 2), p. 118; et Warburg (ibid.), WIA, ZK 041/021148-149.

5. Warburg (ibid), WIA, ZK 041/021148-149. Semper mentionne un 'serpent d'eau' mais pas un "ver" dans son essai de 1856 (ibid., p. 119); il est fait cependant référence aux vers dans le "Prolegomena" de son texte Der Stil. Voir Semper, Der Stil (1860), p. xxxvii. Il se peut que Warburg ait synthétisé sa lecture des deux textes, car certaines parties de la deuxième section théorique du cours sempérien de 1856 sur la parure se rapportent aux sections du "Prolegomena".

6. Semper, "Über die formelle Gesetzmässigkeit des Schmuckes" (voir note 2), p. 101; et Warburg (ibid.), WIA, ZK 041/021141.

7. Alexander von Humboldt, Kosmos: Entwurf einer physischen Weltbeschreibung (Stuttgart et Tübingen: Cotta, 1845-1862), 5 volumes. Le Kosmos d'Humboldt est sur la liste des livres que Semper a emmené avec lui à Londres. Voir les copies des listes de livres de Semper dans Wolfgang Hermann Papers, Collections Spéciales, Getty Research Institute.

8. Johann Gottlob Schneider, Griechisch-Deutsches Wörterbuch (Leipzig: Hahn'sche Verlag, 1819), vol. 1, p. 785. Sur la liste de livres de Semper.

9. Franz Passow, Handwörterbuch der griechischen Sprache (Leipzig: Friedrich Christian Vogel, 1831), vol. 1, p. 1333.

10. Concernant l'aspect "cosmique" du travail de Semper, voir aussi de Werner Oechslin, “ “...bei furchtloser Konsequenz (die nicht jedermanns Sache ist)...” Prolegomena zu einem verbesserten Verstädnis des Semper'schen Kosmos" dans Gottfried Semper 1803-1879: Architectektur und Wissenschaft, édité par Winfried Nerdinger et Werner Oechslin (Munich-Zurich: Prestel-gta, 2003), pp. 52- 
11. Sur les queues de comètes, voir Semper, Der Stil (1860-63), Vol. 1, p. xxxvi; (see note 1). Sur les projectiles anciens, voir Semper, Über die bleiernen Schleudergeschosse der Alten (Frankfurt: Verlag für Kunst und Wissenschaft, 1859).

12. Pour la relation entre le travail de Semper et la théorie ethnologique, voir H.F. Mallgrave, "Gustav Klemm and Gottfried Semper: The Meeting of Ethnological and Architectural Theory," RES: Anthropology and Aesthetics 9 (Spring 1985): 68-79; et "Semper, Klemm, e l'etnografia-Semper, Klemm, and ethnography," Lotus international, no. 109 (2001): 118-131.

13. Gottfried Semper, Wissenschaft, Industrie und Kunst: Vorschläge zur Anregung nationalen Kunstgefühles bei dem Schlusse der Londoner Industrie-Austellung (Brunswick: Vieweg und Sohn, 1852). Traduction anglaise par H. Mallgrave et Wolfgang Herrmann, dans Gottfried Semper: The Four Elements of Architecture and OtherWritings (New York: Cambridge University Press, 1988).

14. Sur le commentaire de Semper sur Vitruvius, y compris ses observations concernant le rapport entre l'architecture et l'astrologie, voir son "Bemerkungen zu des M. Vitruvius Pollio zehn Bücher der Baukunst," dans Kleine Schriften, ed. Manfred et Hans Semper (Berlin and Stuttgart: Spemann, 1884), pp. 191-212.

15. Sur l'ethnographie des Botocudos au début du XIXe siècle, voir les mémoires de Maximilian Wied-Neuwied de 1815-1817, Reise nach Brasilien (ca. 1825), ed. Wolfgang Joost (Leipzig: Graphische Werke Zwickau, 1987).

16. “ [d]ie Botokuden durchboren ihre Unterlippen und stecken grosse hölzerne Knebel, Knochen, Muscheln oder Aenliches in den Einschnitt, wodurch die Lippe tief heruntergezogen und auf schreckbare Weise verlängert wird." Semper (voir note 2), p. 102.

17. Semper, "Über die formelle Gesetzmässigkeit des Schmuckes” (voir note 2), p. 103.

18. Semper, "Über die formelle Gesetzmässigkeit des Schmuckes" (voir note 2), p. 117.

19. Sur les paramètres linguistiques des études sur l'ornement par Semper et Riegl, voir Debra Schafter, "Ornament and Language," dans The Order of Ornament, The Structure of Style: Theoretical Foundations of Modern Art and Architecture (Cambridge: Cambridge University Press, 2003), pp. 60-102.

20. Semper, “Über die formelle Gesetzmässigkeit des Schmuckes” (voir note 2), p. 113.

21. Semper, "Über die formelle Gesetzmässigkeit des Schmuckes" (voir note 2), p. 106. Dans son analyse de la conférence 1856 de Semper, Mallgrave relève que Semper emprunte les termes "macrocosmique" et "microcosmique" aux écrits d'Adolf Zeising sur l'esthétique et les théories de la proportion humaine, même si l'architecte s'est fortement opposé à l'esthétique idéaliste de Zeising. Voir H.F. Mallgrave, Gottfried Semper, Architect of the Nineteenth Century (New Haven: Yale Univ. Press, 1996), p. 271.

22. Semper, “Über die formelle Gesetzmässigkeit des Schmuckes” (voir note 2), pp. 107-108.

23. Ibid., p. 106.

24. Ibid., p. 109.

25. Ibid., p. 112

26. Ibid., p. 109.

27. Pour des exemples similaires, voir Hubert Stierling, Der Silberschmuck der Nordseeküste (Neumünster in Holstein: Karl Wachholtz, 1935).

28. Semper, "Über die formelle Gesetzmässigkeit des Schmuckes" (voir note 2), p. 113

29. Ibid., pp. 113-114.

30. Pour une description antérieure de ces reliefs, voir Austen Henry Layard, Nineveh and its remains (London, J. Murray, 1849).

31. Pour l'association du Kosmos avec des formes d'organisation militaire, voir l'analyse étymologique du mot par Claus Haebler, "KOSMOS: Eine etymologisch-wortgeschichtliche Untersuchung," dans Archiv für Begriffsgeschichte 11 (1967): 101-118.

32. Semper, "Über die formelle Gesetzmässigkeit des Schmuckes" (voir note 2), p. 114; et Warburg (voir note 3), WIA ZK 041/021145. 
33. Gottfried Semper, dessin, archives Semper, gta, ETH, Zurich. 20-0212-8. Dans son dessin Semper copie un bas-relief néo-attique de la fin du deuxième siècle avant J.C. (d'après un original de l'œuvre du sculpteur Kallimachos, fin du cinquième siècle avant J.C. à Rome.

34. Tafel 6 "Raub, Opfer, Opfernde Mänade..." dans Aby Warburg, Der Bilderatlas Mnemosyne herausgegeben von Martin Warnke under Mitarbeit von Claudia Brink (Berlin: Akademie Verlag, 2000), p. 24-25.

35. Warburg (ibid.), WIA, ZK 041/021155.

36. La connexion entre Warburg et Semper a jusqu'ici été très peu étudiée. Ernst Gombrich cite brièvement la lecture de Warburg de la conférence de Semper de 1856, pendant la période au cours de laquelle il a écrit sa thèse. Voir Ernst Gombrich, Aby Warburg: An Intellectual Biography (Chicago: University of Chicago Press, 1986), p. 80. Gombrich accorde plus d'attention à l'essai de Semper dans sa propre étude sur l'ornementation, où il relie les "spéculations a priori" de Semper à Goethe et Aristote. Voir E. H. Gombrich, The Sense of Order: A study in the psychology of decorative art (London: Phaidon, 1994), pp. 48-49.

37. “9. Kampf gegen die Philister.-[F]latternde Gewänder (entgegengesetzen Richtungen). Volute im Helm." WIA, III.33.2.8 Aby Warburg, notes de cours "Von Nicolo Pisano bis Michelangelo. Vorlesung von Prof. A. Schmarsow gehalten im Kunsthistorischen Institut zu Florenz, W-S 88/89,“" (Décembre 10-14, 1888), p. 67. Pour le cours publié de Schmarsow sur les portes de Ghiberti, voir August Schmarsow, Ghibertis Kompositionsgesetze an der Nordtür des Florentiner Baptisteriums (Leipzig: B.G. Teubner,1899). Sur le même thème, voir Georges DidiHuberman, "Bewegende Bewegungen: Die Schleier der Ninfa nach Aby Warburg" dans Ikonologie des Zwischenraums: der Schleier als Medium und Metapher édité par J. Endres, B. Wittmann und G. Wolf (Paderborn: Fink, 2005), pp. 339-40.

38. Warburg (voir note 4), WIA, ZK 041/021140

39. Aby Warburg, Dissertation Handexemplar (épreuves imprimées de la thèse publiée avec des annotations manuscrites d'Aby Warburg), WIA III. 40.1.1.2, p. 10.

40. Pour la thèse publiée, voir Aby Warburg, Sandro Botticellis "Geburt der Venus" und "Frühling" : eine Untersuchung über die Vorstellungen von der Antike in der italienischen Frührenaissance (Hamburg et Leipzig: Leopolod Voss, 1893). La thèse, y compris une version révisée comprenant des addenda de Warburg, a été publiée dans Aby Warburg, Gesammelte Schriften. Die Erneuerung der heidnischen Antike: Kulturwissenschaftliche Beiträge zur Geschichte der Europäischen Renaissance, ed. Gertrund Bing avec la collaboration de Fritz Rougement (Leipzig-Berlin: Taubner, 1932), pp. 1-60 et 302-328.

41. Par exemple, Horst Bredekamp, "Fallende Flammen," dans Botticelli Primavera: Florenz als Garten der Venus (Frankfurt am Main: Fischer Taschenbuch Verlag, 1988), pp. 40-46.

42. à partir de l'essai suivant de Warburg sur l'artiste, "Sandro Botticelli," dans Warburg, Gesammelte Schriften (see note 38), Vol. 1, pp. 61-68.

43. „Beim Schmuck steht der dargestellte Ausdruck in keinem organischen Verhältnis zum Träger, insofern als die Oberfläche nicht der natürliche Erfolg der augenblicklichen Kraft u.[nd] des Willensverhältnisses des Trägers ist." Aby Warburg, Grundlegende Bruchstücke zu einer monistische Kunstpsychologie, WIA III.43.1.3, 43, aphorisme numéro 100, en date du 6 Octobre, 1890. 44. Aby Warburg, Thèse sur Botticelli, versions préliminaires WIA III. 38.1.2. Warburg utilise le terme similaire "la dynamisation d'autres formes" (dynamisierende Zusatzformen) dans les aphorismes philosophiques ajoutés plus tard à sa thèse et intitulés "Quatre thèses." Warburg, Gesammelte Schriften (voir note 38), p. 58.

45. "Bewegte Gewandmotive in der Florentiner Kunst des Quattrocento. (A) 1. H. des Quattrocento. (1) Bewegung des Gewandes ohne Motivierung durch den Körperbau. (2) Bewegung des Gewandes den Natürlichen Voraussetzungen zuwider. ... (B) 2.H. 1450-1500. (1) Bewegung des Gewandes unter gleichzeitiger Bewegung des Körpers. (2) Bew. D. Gew. D. natürl. Verlauf zuwider." Quoted in 
Gombrich, Aby Warburg (see note 34), p. 48. Pour l'original voir WIA, ZK Ae. Aphorismen 27 mars, 1889.

46. "Per queto genere di ornamento il G. Semper ha trovato la parola addatta "Richtungschmuck" [sic]."Aby Warburg, "I costumi teatrali per gli intermezzi del 1589. I disegni di Bernardo Buontalenti e il libro di Conti di Emilio de' Cavalieri” dans Gessamelte Schriften, Vol. 1, p, 292, n.2.

47. J.Matthias, Der Meschliche Schmuck. Form, Farbe und Anwendung. Ein Beitrag zur Bildung des Geschmacks in häuslichen und gewerblichen Kreisen (Leipzig: Verlag von H.Haessel, 1870).

48. "[Er] will der Schönheitssinn d.[er] Hausfrau wecken; basiert auf Semper ohne zu citieren." Aby Warburg, notes bibliographiques, "J.Matthias. Der menschliche Schmuck" dans Zettelkasten marqué Gerät-Tracht, WIA ZK 29A 010/004528.

49. Voir par exemple le catalogue d'exposition, Gold und Silberschmuck nach Originalen der Strassburger historischen Schmuck-Ausstellung von 1904, ed. Robert Forrer (Strassburg: Ludolf Beust, 1905). Pour une analyse complète de l'ornement dans les cultures différentes, voir Völkerschmuck, ed. Martin Gerlach, introduction et légendes par Michael Haberlandt (feu le conservateur du Naturhistorisches Museum de Viennes) (Wien-Leipizg: Verlag von Gerlach \& Wiedling, 1906). Pour une étude plus centrée sur la parure européenne, voir Ernst Bassermann-Jordan, Der Schmuck (Leipzig: Klinkhardt \& Biermann, 1909). Pour une histoire de la joaillerie, voir Hermann Barth, Das Geschmeide: Schmuck und Edelsteinkunde, vol.1, Die Geschichte des Schmuckes et vol. 2, Das Material des Schmuckes (Berlin: Verlagsbuchhandlung Alfred Schall, 1903).

50. Emil Selenka, Der Schmuck des Menschen (Berlin: VITA Deutsches Verlagshaus, 1900). Voir la note bibliographique "Emil Selenka, Der Schmuck des Menschen, Berlin," dans Gerät -Tracht, WIA ZK 29A 010/004526. Les notes de Warburg comprennent d'autres références à Selenka, tels que ses articles sur le mime et la physionomie, "Ueber die Mimik" et "Die Sprache des menschliches Angesichts" publiés par la société anthropologique à Munich en 1890. Aby Warburg, Ausdruckskunde WIA, ZK 001/000070 et 000074.

51. Emil Selenka et Leonore Selenka, Sonnige Welten: Ostasiatische Reise-Skizzen (Borneo, Java, Sumatra, Vorderindien, Ceylon, Japan) (ca.1896) 3rd rev. ed. (Berlin: C.W. Kreidel's Verlag, 1925).

52. Pour une analyse plus complète du texte et de sa relation avec Semper, consultez mon article "World ornament: The legacy of Gottfried Semper's 1856 lecture on adornment" dans RES: Anthropology and Aesthetics No. 57/58, pp. 309- 329.

53. "Ich habe so eine Idee! ...die ganze Frage (der Genesis) der Ornamentik auf eine ganz breite Basis zu stellen mit Einbeziehung des ethnographischen Materials. Deshalb gehe ich wahrscheinlich Anfang nächste Woche westwärts, um dort die Pueblo Indianer in New Mexico zu besuchen. Aber das ist alles noch im Nebel." WIA, General Correspondence, Aby Warburg à Mary Herz le 8 novembre, 1895. Salvatore Salvatore Settis, mentionne aussi que le point d'intérêt initial était l'ornement. Settis, Salvatore."Kunstgeschichte als vergleichende Kulturwissenschaft: Aby Warburg, die Pueblo-Indianer und das Nachleben der Antike.” Dans Künstlerischer Austausch (Artistic Exchange): Akten des XXVIII. Internationalen Kongresses für Kunstgeschichte Berlin, 15.-20. Juli 1992. Ed. Thomas W. Gaehtgens. Vol. 1.Berlin: Akademie Verlag, 1993.139-58.

54. Voir les fichiers dans Zettelkasten marqués "Americana”, WIA ZK No. 40. Pour la collection ethnographique des objets pueblos et dessins donnés au Museum für Völkerkunde à Hambourg, voir Benedetta Cestelli Guidi "Aby Warbug collezionista" et "La collection Pueblo d'Aby Warburg, 1895-1896."

55. WIA ZK 040/020761-763.

56. Voir les notes sur "Pueblo Ind.[ianer] Ornamentik" in WIA ZK, 040/020693-97. Pour un compte-rendu contemporain des motifs ornementaux analogues en Amérique du nord, voir Hjalmar Stolpe, "Studies in American Ornamentation: A contribution to the Biology of Ornament" dans Collected Essays in Ornamental Art (Stockholm: Aftonbladets tryckeri, 1927) 72-112. 
57. Voir les dessins en couleur avec des symboles zuni, datés de 1885 dans Bandelier, Adolph. A History of the Southwest. A study of the civilization and conversion of the Indians in Southwestern United States and Northwestern Mexico from the earliest times to 1700. Ed. Ernest J. Burrus. Vol. I, supplément. (Vatican: 1969 et 1987) 117 et pl. II.

58. Voir les esquisses de Warburg, WIA ZK, 040/020727-29 [illustration 728].

59. Sur Umfangsbestimmung ainsi que sur l'horizon épistémologique et Kosmos, voir Bernd Villhauer, Aby Warburgs Theorie der Kultur: Detail und Sinnhorizont (Berlin: Akademie Verlag, 2002), 68-71.

60. Sur l'interdépendance entre les modes de pensée allégoriques et symboliques, et pour leur relation aux images cosmiques dans les traditions littéraires et iconographiques occidentales, voir le chapitre "The Cosmic Image" dans l'étude classique d'Angus Fletcher, Allegory: The Theory of a Symbolic Mode. Cornell UP, 1964, 70-146.

61. La relation entre l'ornement, le symbolisme et l'idée d'Umfangsbestimmung est analysée dans le texte de Warburg "Symbolismus als Umfangsbestimmung, » un appendice de son «Fragments pour une psychologie moniste de l'art " qu'il avait ébauché lorsqu'il était en Californie en 1896 mais auquel il a fait de nombreux ajouts par la suite (WIA III, 45.2). Dans ce court passage esthétique, Warburg se base considérablement sur Semper, tout particulièrement sur les termes utilisés par Semper dans sa conférence de 1856 sur Schmuck. Par exemple, près du terme «Umfangsbestimmung » décrit ici comme «Umschreibung » (définition périphérique) Warburg emploie les termes "Richtungsbestimmung » et "Längsrichtungsbestimmung " (WIA III, 45.2, page 15). Warburg se sert également de la paire de termes opposés « differenzirende Dynamik» et «schematisirende Statik» (32) qu'il représente par deux flèches pointant vers des directions opposées; comme nous l'avons vu dans les notes de Warburg et dans les feuillets manuscrits rajoutés à la version publiée de sa thèse, « Dynamique et statique " sont deux science naturelles qui, selon Semper, formaient la base de la science esthétique. Dans ce qui se révèle être l'appendice explicatif d'un diagramme conceptuel, Warburg explique que la lettre « $\mathrm{Z}$ » connote le « differenzierte Zeichen » (le signe différentiel) et la lettre « $\mathrm{M}$ » la « appercipierende Masse » (masse saisissante) : Warburg a utilisé les lettres « $M$ » et « $Z$ » dans sa petite esquisse des axes cardinaux de croissance dans une des pages qui contient ses notes de lecture sur l'essai de Semper de 1856 (fig.2). Cependant, il faut remarquer comment l'historien de l'art convertit les termes scientifiques utilisés par l'architecte en idéogrammes personnels afin de construire son propre système symbolique. Pour une version publiée du texte de Warburg, voir «Symbolismus als Umfangbestimmung » dans Aby Warburg, Werke in Einem Band ed. Martin Treml, Sigrid Weigel, et Perdita Ladwig (Berlin: Suhrkamp, 2010), pp. 615-628.

62. Concernant la lecture de Warburg de l'oeuvre Carlyle, Sartor Resartus, voir Ernst Gombrich, Aby Warburg: An Intellectual Biography, (Chicago: The University of Chicago Press, 1986), pp. 14, 75 ; et Bernd Villhauer, Aby Warburg's Theorie der Kultur: Detail und Sinnhorizont, (Berlin: Akademie Verlag, 2002), pp. 39-44.

63. Le schéma préliminaire correspond à l'aphorisme numéro 399, daté du 13 avril, 1900 (Florence), qui comprend à la fois les termes "Umfangsbest.[immung]" et "Umfang," soit comme "Spiel - Umfang" ou "Klarer Umfang", ainsi que les termes "Schmuck" et "sym.[bolisches] objekt." WIA ZK Ae.[sthetik] Aphorismen and Warburg, Grundlegende Bruchstücke, WIA, III.43.2.1.

64. La photo est publiée dans Photographs at the Frontier: Aby Warburg in America 1895-1896, édité par Benedetta Cestelli Guidi et Nicholas Mann (London: Holberton / Warburg Institute, 1998) p. 91 , plate 21.

65. Selenka, Der Schmuck des Menschen, (voir note 50), pp. 69-70.

66. Aby Warburg, Schlangenritual. Ein Reisebericht, Nachwort von Ulrich Raulff (Berlin: Wagenbach, 1996), p. 56. 
INDEX

Mots-clés : Modèles, analogie, harmonies fragmentaires, imagination, correspondances, ethnographie, Botocudos

\section{AUTEURS}

SPYROS PAPAPETROS

Historien de l'art et de l'architecture enseigne à la faculté d'Architecture de l'université de Princeton. Il s'intéresse notamment aux intersections entre architecture et arts figuratifs et à la relation entre architecture, psychanalyse et histoire des esthétiques psychologiques. Il est l'auteur de On the Animation of the Inorganic: Art, Architecture, and the Extension of Life (University of Chicago Press, 2011) 